\title{
Soft corals (Octocorallia: Alcyonacea) of the southern Ryukyu Archipelago: The families Tubiporidae, Clavulariidae, Alcyoniidae and Briareidae
}

\author{
Y. Benayahu \\ Department of Zoology, George S. Wise Faculty of Life Sciences, Tel Aviv \\ University, Ramat Aviv, Tel Aviv 69978, Israel. \\ E-mail address: yehudab@tauex.tau.ac.il \\ Phone: 972-3-6409090 \\ F a x: 972-3-6409403
}

\begin{abstract}
Species composition of soft corals from the Yaeyama and Miyako island groups, of Sakishima Is., located at the southern part of the Ryukyu Archipelago were studied during 1992 and 1993. SCUBA collections were carried out at 18 sites around the islands down to $30 \mathrm{~m}$. The collection, comprising approximately 200 specimens, yielded 45 species. The survey established two new species: Sinularia higai and S. tanakai, and in addition revealed 14 new zoogeographical records. Among the latter are Protodendron and Rhytisma species, which are the first records of these genera in the Ryukyu Archipelago. The obtained results along with a previous survey on Sesoko Is. show the genus Sinularia have the highest species diversity on the reefs, comprising $33-45 \%$ of total species number. Synthesizing the existing data for the south Ryukyu Archipelago yielded a total of 56 soft coral species (11 genera) of the families Clavulariidae, Tubiporidae, Alcyoniidae and Briareidae.

Since the survey was conducted prior to the recent mass coral bleaching event (1998) in the region, it is of prime importance to monitor changes in soft coral species diversity and abundance there. Such data will indicate possible shifts in benthic community structure on the reefs and the rate of recovery.
\end{abstract}

Key words: Octocorallia, Japan, Ryukyu Archipelago, taxonomy, coral reefs, species diversity

\section{INTRODUCTION}

The history of systematic studies on the octocoral fauna of the Japanese Archipelago has been reviewed by Imahara (1996). Soft corals inhabiting the reefs of various islands have been the subject of several studies. The work of Utinomi (1976a,b; 1977a,b) dealt with specimens collected mainly from the shallow reefs of Okinawa, and that of Imahara (1991), mainly from Kerama and Iriomote Islands. More recently, Williams (1997) reported a new genus from approximately $60 \mathrm{~m}$ in depth off Okinawa. In an earlier study by Benayahu (1995), conducted on the coral reefs of Sesoko Island, 39 species of the families Clavulariidae, Alcyoniidae and Xeniidae were listed, among which two were new species and 20 new zoogeographical records.

The present paper is thus the second in a series aimed at an in depth investigation of the soft corals (Octocorallia, Alcyonacea) of the reefs of the Ryukyu Archipelago, Japan. It examines the soft coral fauna (Alcyonacea) of the families Tubiporidae, Clavulariidae, Alcyoniidae and Briareidae collected from the Sakishima Is. (Yaeyama and Miyako Groups), southern Ryukyu Archipelago. A systematic list is presented with annotations on their distribution, habitat and abundance on 
the studied Islands. For some of the more common species the study also presents remarks on their underwater characteristics.

\section{METHODS}

Collecting trips were conducted in the Yaeyama and Miyako island groups, of the Sakishima Is., located at the southern part of the Ryukyu Archipelago (Fig. 1). The first trip was carried out during November 1992 to Ishigaki, Iriomote, Kohama, Miyako, Shimoji and Yonaguni Islands, and the second during July 1993 to Hatoma, Ishigaki and Yonaguni Islands. Some additional material was collected during 1996 by Dr. Tetsuo Iwagawa and Mr. Kaoru Takemura from Ishigaki Island and was also included in the study. The studied reefs are listed below, totalling 18 sites, with their coordinates:



Fig 1. a. Map of the study area with the collecting sites on the different islands indicated by numbers: b. Miyako Islands: Miyako Is. 1. Hisamatsu Channel; Shimoji Is., 1. Mao Cave, 2. Kuro Buoy: c, d. Yaeyama Islands: Yonaguni Is. 1. Higawa, 2. Nurugan, 3. Akatsuchi, 4. Off Danno, 5. Umabana, 6. Off Sonai; Ishigaki Is. 1. Off Taketomi, 2. Masa-guchi, 3. Yarabu, 4. Ibaruma, 5. Hirakubo Cape; Kohama Is. 1. Yonara; Iriomote Is. 1. Kanokawa; Hatoma Is. 1. Southern side, 2. Northern side. 
Yaeyama Islands:

Yonaguni Is.

1. Higawa $24^{\circ} 26^{\prime} \mathrm{N} ; 122^{\circ} 59^{\prime} \mathrm{E}$

2. Nurugan $24^{\circ} 26^{\prime} \mathrm{N} ; 122^{\circ} 57^{\prime} \mathrm{E}$

3. Akatsuchi $24^{\circ} 26^{\prime} \mathrm{N} ; 122^{\circ} 56^{\prime} \mathrm{E}$

4. Off Danno $24^{0} 27^{\prime} \mathrm{N} ; 122^{\circ} 57^{\prime} \mathrm{E}$

5. Umabana $24^{\circ} 28^{\prime} \mathrm{N} ; 122^{\circ} 58^{\prime} \mathrm{E}$

6. Off Sonai $24^{0} 28^{\prime} \mathrm{N} ; 122^{\circ} 59^{\prime} \mathrm{E}$

Ishigaki Is.

1. Off Taketomi $24^{\circ} 20^{\prime} \mathrm{N} ; 124^{\circ} 05^{\prime} \mathrm{E}$

2. Masa-guchi $24^{\circ} 20^{\prime} \mathrm{N} ; 124^{\circ} 04^{\prime} \mathrm{E}$

3. Yarabu Cape $24^{\circ} 25^{\prime} \mathrm{N} ; 124^{\circ} 04^{\prime} \mathrm{E}$

4. Ibaruma $24^{\circ} 33^{\prime} \mathrm{N} ; 124^{\circ} 17^{\prime} \mathrm{E}$

5. Hirakubo Cape $24^{\circ} 37^{\prime} \mathrm{N} ; 124^{\circ} 19^{\prime} \mathrm{E}$

Kohama Is.

1. Yonara $24^{\circ} 19^{\prime} \mathrm{N} ; 123^{\circ} 57^{\prime} \mathrm{E}$

Iriomote Is.

1. Kanokawa $24^{0} 17^{\prime} \mathrm{N} ; 123^{\circ} 44^{\prime} \mathrm{E}$

Hatoma Is.

1. Southern side $24^{\circ} 27^{\prime} \mathrm{N} ; 123^{\circ} 49^{\prime} \mathrm{E}$

2. Northern side $24^{\circ} 28^{\prime} \mathrm{N} ; 123^{\circ} 49^{\prime} \mathrm{E}$

\section{Miyako Islands}

Miyako Is.

1. Hisamatsu Channel $24^{\circ} 46^{\prime} \mathrm{N} ; 125^{\circ} 14^{\prime} \mathrm{E}$ Shimoji Is.

1. Mao Cave $24^{\circ} 49^{\prime} \mathrm{N} ; 125^{\circ} 08^{\prime} \mathrm{E}$

2. Kuro Buoy $24^{\circ} 47^{\prime} \mathrm{N} ; 125^{\circ} 13^{\prime} \mathrm{E}$

The collection sites were reached by boat and a variety of habitats were surveyed in detail by SCUBA diving on onshore reefs, in lagoons and on deeper reefs to $30 \mathrm{~m}$ depth. Approximately 200 soft coral samples of the currently studied families were collected, comprising the full variety of species found on the reefs. At each site underwater abundance-estimates of the different species were visually made and divided into four categories: rare, sporadic, abundant and dominant (see also Benayahu 1995). Prior to collection, most of the colonies were photographed underwater, using a Nikonos $\mathrm{V}$ camera with a close-up attachment. Samples were fixed in $4 \%$ formalin in seawater, rinsed in fresh water after 24 hours, and then transferred to $70 \%$ ethyl alcohol. Sclerites were obtained by dissolving the tissues in $10 \%$ sodium hypochlorite. Identification of the alcyoniid species in the collection was in a great part facilitated by comparisons with permanent sclerite-preparations from type material kept in the Zoological Museum, Department of Zoology, Tel Aviv University, Israel (ZMTAU) and at the National Natuurhistorisch Museum, formerly Rijksmuseum van Natuurlijke Historie, Leiden, The Netherlands. Scanning electron microscopy of the sclerites followed the methodology described by Benayahu (1995). All material is kept in the Zoological Museum, Department of Zoology, Tel Aviv University, Israel. Specimens of the family Briareidae were kindly identified by L.P. van Ofwegen, National Museum of Natural History, Leiden, The Netherlands, and upon his agreement also included in this study. Members of the families Nephtheidae, Nidaliidae and Xeniidae were also collected and are still being examined.

\section{RESULTS}

The examined material yielded 45 species, of which, two are new species and 14 are new records from the Ryukyu Archipelago.

\section{LIST OF THE SPECIES}

Clavulariidae Hickson, 1894

Genus Clavularia Blainville, 1830

Clavularia inflata Schenk, 1896

Clavularia viridis (Quoy \& Gaimard, 1833)

Tubiporidae Ehrenberg, 1828

Genus Tubipora Linnaeus, 1758

Tubipora musica Linnaeus, 1758

Alcyoniidae Lamouroux, 1812

Genus Cladiella Gray, 1869

Cladiella australis (Macfadyen, 1936)

Cladiella pachyclados (Ehrenberg, 1834)

Genus Eleutherobia Putter, 1990

Eleutherobia grayi (Thomson \& Dean, 1931)

Genus Klyxum Alderslade, 2000

Klyxum simplex (Thomson \& Dean, 1931)

Klyxum utinomii (Verseveldt, 1971)

Genus Lobophytum von Marenzeller, 1886

Lobophytum batarum Moser, 1919

Lobophytum crassum von Marenzeller, 1886

Lobophytum durum Tixier-Durivault, 1957

Lobophytum pauciflorum (Ehrenberg, 1834)

Lobophytum venustum Tixier-Durivault, 1957

Genus Protodendron Thomson \& Dean, 1931

Protodendron repens Thomson \& Henderson, 1906

Genus Rhytisma Alderslade, 2000

Rhytisma fulvum fulvum (Forskal, 1775)

Genus Sarcophyton Lesson, 1834

Sarcophyton crassocaule Moser, 1919

Sarcophyton ehrenbergi von Marenzeller, 1886

Sarcophyton glaucum (Quoy \& Gaimard, 1833)

Sarcophyton infundibuliforme Tixier-Durivault, 1958

Sarcophyton roseum Pratt, 1903

Sarcophyton tenuispiculatum Thomson \& Dean, 1931

Sarcophyton trocheliophorum von Marenzeller, 1886

Genus Sinularia May, 1898 
Sinularia brassica May, 1898

Sinularia ceramensis Verseveldt, 1977

Sinularia compressa Tixier-Durivault, 1945

Sinularia erecta Tixier-Durivault, 1945

Sinularia flexibilis (Quoy \& Gaimard, 1833)

Sinularia gibberosa Tixier-Durivault, 1970

Sinularia gravis Tixier-Durivault, 1970

Sinularia heterospiculata Verseveldt, 1970

Sinularia higai spec. nov.

Sinularia humesi Verseveldt, 1968

Sinularia leptoclados (Ehrenberg, 1834)

Sinularia lochmodes Kolonko, 1926

Sinularia mollis Kolonko, 1926

Sinularia numerosa Tixier-Durivault, 1970

Sinularia ovispiculata Tixier-Durivault, 1970

Sinularia parva Tixier-Durivault, 1970

Sinularia polydactyla (Ehrenberg, 1834)

Sinularia querciformis (Pratt, 1903)

Sinularia tanakai spec. nov.

Sinularia variabilis Tixier-Durivault, 1945

Sinularia vrijmoethi Verseveldt, 1971

Briareidae Blainville, 1830

Genus Briareum Blainville, 1830

Briareum excavatum (Nutting, 1911)

Briareum violacea (Quoy \& Gaimard, 1833)

\section{DESCRIPTION OF THE MATERIAL}

Most of the identified soft corals have been adequately described elsewhere, and therefore relevant references are given in the text follow ${ }^{-}$ ing the abbreviated synonymies. A survey of the species with remarks on their distribution in the study area is presented below.

Family Clavulariidae Hickson, 1894

Genus Clavularia Blainville, 1830

Clavularia inflata Schenk, 1896

Clavularia inflata Schenk, 1896; Imahara 1991: 59-60; for further references see Imahara 1996: 19; van Ofwegen 1996: 208 (listed only).

Local occurrence. Kohama Is., Yonara, $1 \mathrm{~m}, 15$ November 1992 (ZMTAU Co 28694, 4 specimens); Hatoma Is., southern side, $5 \mathrm{~m}$, 3 July 1993 (ZMTAU Co 28680, 2 specimens); Yonaguni Is., Higawa, reef flat, 9 November 1992 (ZMTAU Co 28677).

Field notes. Sporadic.

Geographical distribution. Ternate, Great Barrier Reef, Indonesia, Philippines, Tokara Is., Formosa, Micronesia, Ryukyu Archipelago, Bismarck Sea.

Clavularia viridis (Quoy \& Gaimard, 1833)
Clavularia viridis (Quoy \& Gaimard, 1833); for synonymy see Thomson \& Dean 1931: 14; for further references see Imahara 1996: 19.

Local occurrence. Ishigaki Is., off Taketomi, 19 m, 14 November 1992 (ZMTAU Co 28682); Kohama Is., 2 m, 15 November 1992 (ZMTAU Co 28695, 2 specimens, Co 28678, 6 specimens), $1 \mathrm{~m}$ (ZMTAU Co 28686); Iriomote Is., 16 m, 16 November 1992 (ZMTAU Co 28696, 2 specimens).

Field notes. Sporadic.

Geographical distribution. New Hebrides, Ryukyu Archipelago, Indonesian Archipelago.

Family Tubiporidae Ehrenberg, 1828 Genus Tubipora Linnaeus, 1758 Tubipora musica Linnaeus, 1758

Tubipora musica Linnaeus, 1758; Verseveldt 1965: 28 (listed only); Benayahu 1993: 5 (listed only); Benayahu \& Schleyer 1996: 4 (listed only); van Ofwegen 1996: 207 (listed only); for further references see Imahara 1996: 19.

Local occurrence. Ishigaki Is., off Taketomi, $10 \mathrm{~m}$, 14 November 1992 (ZMTAU Co 28699), Yarabu Cape, 20 m, 17 November, 1992 (ZMTAU Co 28711, 2 specimens); Hatoma Is., northern side, 10 m, 3 July 1993 (ZMTAU Co 28712); Kohama Is., Yonara, $1 \mathrm{~m}, 15$ November 1992 (ZMTAU Co 28717); Yonaguni Is., Nurugan, 12 m, 11 November 1992 (ZMTAU 28710, 3 specimens); $18 \mathrm{~m}$ (ZMTAU Co 28709), Higawa, 10 m, 10 November 1992 (ZMTAU Co 28713).

Field notes. Abundant.

Geographical distribution. Widespread in the IndoWest Pacific reefs.

Family Alcyoniidae Lamouroux, 1812 Genus Cladiella Gray, 1869 Cladiella australis (Macfadyen, 1936)

Cladiella australis (Macfadyen, 1936); for synonymy see Benayahu \& Schleyer 1996: 7-12.

Local occurrence. Ishigaki Is., off Taketomi, $10 \mathrm{~m}$, 14 November 1992 (ZMTAU Co 28700).

Field notes. Rare.

Geographical distribution. Australia, Fiji Islands, Vietnam, Tanzania, Mozambique, Ryukyu Archipelago.

Cladiella pachyclados (Klunzinger, 1877)

Cladiella pachyclados (Klunzinger, 1877); for synonymy see Verseveldt 1971: 9-10; for further 
references see Imahara 1996: 20 and Benayahu 1997: 210 .

Local occurrence. Yonaguni Is., Higawa, $6 \mathrm{~m}, 10$ November 1992 (ZMTAU Co 28690), off Sonai, 10 m, 12 November 1992 (ZMTAU Co 28688, 2 specimens); Hatoma Is., southern side, $6 \mathrm{~m}, 3$ July 1993 (ZMTAU Co 28692); Ishigaki Is., Hirakubo cape, 14 m, 4 July 1993 (ZMTAU Co 28691); Miyako Is., Kuro Buoy, 11 m, 19 November 1992 (ZMTAU Co 28702, 2 specimens).

Field notes. Sporadic.

Geographical distribution. Widespread in the IndoWest Pacific reefs.

Genus Eleutherobia Putter, 1900 Eleutherobia grayi (Thomson \& Dean, 1931)

Eleutherobia grayi (Thomson \& Dean, 1931); for synonymy and taxonomic assessment see Williams 2001: 209-225.

Local occurrence. Miyako Is., Kuro Buoy, 25 m, 19 November 1992 (ZMTAU Co 30569).

Field notes. Rare.

Geographical distribution. New Guinea, Indonesian Archipelago, Ryukyu Archipelago.

Genus Klyxum Alderslade, 2000 Klyxum simplex (Thomson \& Dean, 1931)

Klyxum simplex (Thomson \& Dean, 1931); for references see Benayahu 1997: 211 and Alderslade 2000: 240-242.

Local occurrence. Ishigaki Is., off Taketomi, $17 \mathrm{~m}$, 14 November 1992 (ZMTAU Co 28698), Hirakubo Cape, 2 m, 4 July 1993 (ZMTAU Co 28687), Yarabu Cape, 24 m, 17 November 1992 (ZMTAU Co 29299), 20 m (ZMTAU Co 29292); Kohama Is., Yonara, 1 m, 15 November 1992; (ZMTAU Co 28701), 2 m (ZMTAU Co 28706, 2 specimens), 15 November 1992: Miyako Is., Kuro Buoy, 7 m, 19 November 1992, (ZMTAU Co 28693, 3 specimens), $10 \mathrm{~m}$ (ZMTAU Co 28714).

Field notes. Sporadic.

Geographical distribution. Bay of Brima, New Caledonia, Madagascar, Ambon (Indonesia), Ryukyu Archipelago, Bismarck Sea, Guam.

\section{Klyxum utinomii (Verseveldt, 1971)}

Klyxum utinomii (Verseveldt), 1971; Verseveldt 1974: 3; 1978: 49 (listed only); van Ofwegen 1996: 207 (listed only) and Alderslade 2000:
240-242.

Local occurrence. Ishigaki Is. off Taketomi, $5 \mathrm{~m}$, 14 November 1992 (ZMTAU Co 28707), $13 \mathrm{~m}$ (ZMTAU Co 28708), Ibaruma, 18 m, 4 July 1993 (ZMTAU Co 28689).

Field notes. Rare. Living colonies are flexible and soft, characterized by dark brown polyps.

Geographical distribution. Madagascar, Red Sea, Guam, Bismarck Sea, a new record for the Ryukyu Archipelago.

Genus Lobophytum von Marenzeller, 1886 Lobophytum batarum Moser, 1919

Lobophytum batarum Moser, 1919; for further references see Imahara 1966: 20 and Benayahu 1997: 212.

Local occurrence. Ishigaki Is., off Taketomi, $10 \mathrm{~m}$, 14 November 1992 (ZMTAU Co 28650); Ibaruma, 16 m, 4 July 1993 (ZMTAU Co 28652, 2 specimens); Iriomote Is., Kanokawa, 22 m, 16 November 1992 (ZMTAU Co 28654); Hatoma Is. northern side, 6m, 3 July 1993 (ZMTAU Cov28649, 3 specimens).

Field notes. Abundant.

Geographical distribution. Philippines, Vietnam, Madagascar, Laccadive Archipelago, Ryukyu Archipelago, Guam.

Lobophytum crassumvVon Marenzeller, 1886

Lobophytum crassum Von Marenzeller, 1886; for synonymy see Verseveldt 1983: 25-32; van Ofwegen \& Vennam 1991: 144 (listed only); 1994: 136 (listed only); van Ofwegen \& Benayahu 1992: 139 (listed only); van Ofwegen 1996: 208 (listed only); Vennam \& van Ofwegen 1996: 438 (listed only); Benayahu 1993: 5 (listed only); 1995: 106 (listed only); Benayahu \& Schleyer 1996: 6 (listed only); for further references see Imahara 1996: 21.

Local occurrence. Ishigaki Is. off Taketomi, $5 \mathrm{~m}$, 14 November 1992 (ZMTAU Co 28721), Yarabu Cape, 6 m, 17 November 1992 (ZMTAU Co 28651), Hirakubo Cape, 9 m, 4 July 1993 (ZMTAU Co 28646); Kohama Is. Yonara, 2 m, 15 November 1992 (ZMTAU Co 28656); Iriomote Is., Kanokawa, $2 \mathrm{~m}, 16$ November 1992 (ZMTAU Co 28445), 3 m (ZMTAU Co 28657); Miyako Is., Hisamatsu Channel, 8 m, 18 November 1992 (ZMTAU Co 28644); Yonaguni Is., Nurugan, 16 m, 11 November 1992 (ZMTAU Co 28647).

Field notes. Dominant. 
Geographical distribution. Widespread in the IndoWest Pacific reefs.

Lobophytum durum Tixier-Durivault, 1956

Lobophytum durum Tixier-Durivault, 1956; for further references see Verseveldt 1983: 45.

Local occurrence. Ishigaki Is., Ibaruma, $16 \mathrm{~m}, 4$ July 1993 (ZMTAU Co 29252).

Field notes. Rare.

Geographical distribution. Vietnam, a new record for the Ryukyu Archipelago.

\section{Lobophytum pauciflorum (Ehrenberg, 1834)}

Lobophytum pauciflorum (Ehrenberg, 1834); for further references see Imahara 1996: 21 and Benayahu 1997: 213.

Local occurrence. Miyako Is., Hisamatsu Channel, 6m, 18 November 1992 (ZMTAU Co 28446, 2 specimens); Kohama Is. Yonara, 4 m, 15 November 1992 (ZMTAU Co 28441), $1 \mathrm{~m}$ (ZMTAU Co 28442, 2 specimens; ZMTAU Co 28444, ZMTAU Co 28653, 2 specimens), 2 m (ZMTAU Co 28643, 2 specimens); Iriomote Is., Kanokawa, 4 m, 16 November 1992 (ZMTAU Co 28443, 3 specimens), $2 \mathrm{~m}$ (ZMTAU Co 28645, 3 specimens); Ishigaki Is., Hirakubo Cape, 9 m, 4 July 1993 (ZMTAU Co 28648), off Taketomi, $6 \mathrm{~m} 14$ November 1992, $6 \mathrm{~m}$ (ZMTAU Co 28658); Yonaguni Is., Higawa, reef flat, 9 November 1992 (ZMTAU Co 28660, 28663, 2 specimens), Umabana, 15 m, 8 July 1993 (ZMTAU Co 28661), off Sonai, 3 m, 12 November 1992 (ZMTAU Co 28662); Hatoma Is., Southern side, $6 \mathrm{~m}$, 3 July 1993 (ZMTAU Co 28664).

Field notes. Dominant. Encrusting colonies with erect lobes, mostly digitiform, sometimes fused at their base, light brown or yellow-brown in color. Geographical distribution. Widespread in the IndoWest Pacific reefs.

\section{Lobophytum venustum Tixier-Durivault, 1957}

Lobophytum venustum Tixier-Durivault, 1957; for further references see Imahara 1996: 21 and Benayahu 1997: 213.

Local occurrence. Yonaguni Is., Higawa, $3 \mathrm{~m}, 10$ November 1992 (ZMTAU Co 28659), off Sonai, 12 m, 12 November 1992 (ZMTAU Co 28641); Hatoma Is., northern side, $8 \mathrm{~m}$, 3 July 1993 (ZMTAU Co 28642, 2 specimens); Ishigaki Is., Ibaruma, 4 July 1993, 7 m (ZMTAU Co 28655, 2 specimens).

Field notes. Sporadic.
Geographical distribution. Widespread in the IndoWest Pacific reefs.

Genus Protodendron Thomson \& Dean, 1931 Protodendron repens (Thomson \& Henderson, 1906)

Protodendron repens (Thomson \& Henderson, 1906); for synonymy see Bayer 1995: 302-303.

Local occurrence. Yonaguni Is., Higawa, $13 \mathrm{~m}, 10$ November 1992 (ZMTAU Co 28681, 10 specimens), Umabana, 15 m, 8 July 1993 (ZMTAU Co 28685, 10 specimens).

Field notes. Rare.

Geographical distribution. Zanzibar, Sangihe Is., Kai Is., Mozambique, a new record for the Ryukyu Archipelago.

Genus Rhytisma Alderslade, 2000

Rhytisma fulvum fulvum (Forskal, 1775)

Rhytisma fulvum fulvum (Forskal, 1775); for synonymy see Verseveldt 1969: 4-7 and Aldersalde 2000: 237.

Local occurrence. Yonaguni Is., Nurugan, $24 \mathrm{~m}$, 11 November 1992 (ZMTAU Co 28715, 5 specimens); $22 \mathrm{~m}$ (ZMTAU Co 28704, 4 specimens); Umabana, 12 m, 6 July 1993 (ZMTAU Co 28705); Iriomote Is., Kanokawa, 4 m, 16 November 1992 (ZMTAU Co 28718, 2 specimens).

Field notes. Rare.

Geographical distribution. Madagascar, Zanzibar, Red Sea, Indonesia, Aldabra, Comoros Ils., Chagos, a new record for the Ryukyu Archipelago.

Genus Sarcophyton Lesson, 1834

Sarcophyton crassocaule Moser, 1919

Sarcophyton crassocaule Moser, 1919; for synonymy see Verseveldt 1982: 27-34; Verseveldt \& Benayahu 1983: 4 (listed only); van Ofwegen 1996: 208 (listed only); Benayahu 1995: 107 (listed only); for further reference see Imahara, 1996: 21.

Local occurrence. Yonaguni Is., Nurugan, $16 \mathrm{~m}, 1$ November 1992 (ZMTAU Co 28666, 2 specimens); 22 m (ZMTAU Co 28673, 2 specimens); Miyako Is., Kuro Buoy, 8 m, 19 November 1992 (ZMTAU Co 28670); Kohama Is., Yonara, $2 \mathrm{~m}, 15$ November 1992 (ZMTAU Co 28671, 2 specimens); Iriomote Is., Kanokawa, 11 m, 16 November 1992 (ZMTAU Co 28676, 4 specimens); Hatoma Is., northern side, $5 \mathrm{~m}$, 3 July 1993 (ZMTAU Co 28675); Ishigaki Is., Hirakubo Cape, 10 m, 5 July 1993 (ZMTAU Co 28667, 2 specimens); $13 \mathrm{~m}$ 
(ZMTAU Co 28672, 2 specimens).

Fied notes. Sporadic.

Geographical distribution. Widespread in the Indo West-Pacific reefs.

Sarcophyton ehrenbergi von Marenzeller, 1886

Sarcophyton ehrenbergi von Marenzeller, 1886; for synonymy see Verseveldt 1982: 40-48; Benayahu 1993: 5 (listed only); 1995: 107 (listed only); van Ofwegen 1996: 208 (listed only); van Ofwegen \& Vennam 1994: 138 (listed only); for further references see Imahara 1996: 21.

Local occurrence. Kohama Is., Yonara, $6 \mathrm{~m}, 15$ November 1992 (ZMTAU Co 28449); Miyako Is., Hisamatsu Channel, 7 m, 18 November 1992 (ZMTAU Co 28457), Kuro Buoy, 11 m, 19 November 1992 (ZMTAU Co 28454); Yonaguni Is., Higawa, reef flat, 9 November 1992 (ZMTAU Co 28460, 28461); Hatoma Is., southern side, 8 m, 3 July 1993 (ZMTAU Co 28674); $7 \mathrm{~m}$ (ZMTAU Co 28665, 2 specimens).

Field notes. Sporadic

Geographical distribution. Widespread in the Indo West-Pacific reefs.

Sarcophyton glaucum (Quoy \& Gaimard, 1883)

Sarcophyton glaucum (Quoy \& Gaimard, 1883); for further references see Imahara 1996: 21 and Benayahu 1997: 214.

Local occurrence. Iriomote Is., Kanokawa, $18 \mathrm{~m}$, 16 November 1992 (ZMTAU Co 28447); Miako Is., Hisamatsu Channel, 6 m, 18 November 1992 (ZMTAU Co 28448); 5 m (ZMTAU Co 28481, 2 specimens); Kuro Buoy, 6 m, 19 November 1992 (ZMTAU Co 28451); Kohama Is., Yonara, 3 m, 15 November 1992 (ZMTAU Co 28452, 2 specimens); $6 \mathrm{~m}$ (ZMTAU Co 28459), 2 m (ZMTAU Co 28485); $7 \mathrm{~m}$ (ZMTAU Co 28487, ZMTAU Co 28491); Yonaguni Is., Higawa, 14 m, 10 November 1992 (ZMTAU Co 28639), Nurugan, 18 m, 11 November 1992 (ZMTAU Co 28462), $15 \mathrm{~m}$ (ZMTAU Co 28482), Umabana, 18 m, 6 July 1993 (ZMTAU Co 28453, 2 specimens), 12 m (ZMTAU Co 28497), off Sonai, $11 \mathrm{~m}, 12$ November 1992 (ZMTAU Co 28483), $12 \mathrm{~m}, 12$ November 1992 (ZMTAU Co 28499), off Danno, 16 m, 6 July 1993 (ZMTAU Co 28488); Hatoma Is., Southern side, $9 \mathrm{~m}, 3$ July 1993 (ZMTAU Co 28455, ZMTAU Co 28668), 8 m (ZMTAU Co 28669); Ishigaki Is., Hirakubo Cape, 2 m, 4 July 1993 (ZMTAU Co 28484, 2 specimens); $12 \mathrm{~m}$ (ZMTAU Co 28489, ZMTAU Co 28496), 10 m, 5 July 1993 (ZMTAU Co 28498, 3 specimens); 12 m (ZMTAU Co 28640), 5 July 1993, Yarabu Cape, 22 m, 17 November 1992 (ZMTAU Co 28486).

Field notes. Dominant. Highly variable with respect to the shape and size of the colony.

Geographical distribution. Widespread in the Indo West-Pacific reefs.

Sarcophyton infundibuliforme Tixier-Durivault, 1958

Sarcophyton infundibuliforme Tixier-Durivault, 1958, for synonymy see Verseveldt 1982: 57-60; van Ofwegen 1996: 208 (listed only); van Ofwegen \& Benayahu 1992: 140 (listed only); Benayahu 1993: 5 (listed only); for further reference see Imahara 1996: 21.

Local occurrence. Ishigaki Is., $1 \mathrm{~m}, 13$ September 1996, leg. K. Takamura \& T. Iwagawa (ZMTAU Co 30015, 30017).

Field notes. Rare. Colonies cup- or funnel-shaped. Geographical distribution. Aldabra Is., Nosy Bay (Madagascar), Ceylon, Bismarck Sea, Tanzania, Sodwana Bay (South Africa), South China Sea, a new record for the Ryukyu Archipelago.

Sarcophyton roseum Pratt, 1903

Sarcophyton roseum Pratt, 1903; for synonymy see Verseveldt 1982: 68-71; van Ofwegen 1996: 208 (listed only).

Local occurrence. Ishigaki Is., off Taketomi, $9 \mathrm{~m}$, 14 November 1992 (ZMTAU Co 28723, 2 specimens); Kohama Is., Yonara, $2 \mathrm{~m}, 15$ November 1992 (ZMTAU Co 28725).

Fied notes. Rare.

Geographical distribution. Maldives, West coast of Australia, Madagascar, Malay Archipelago, Bismarck Sea, a new record for the Ryukyu Archipelago.

Sarcophyton tenuispiculatum Thomson \& Dean, 1931

Sarcophyton tenuispiculatum Thomson \& Dean, 1931; for synonymy see Verseveldt 1982: 80.

Local occurrence. Yonaguni Is., Nurugan, 23 m, 11 November 1992 (ZMTAU Co 28480).

Fied notes. Rare.

Geographical distribution. Djampeah (Flores Sea), New Caledonia, a new record for the Ryukyu Archipelago.

Sarcophyton trocheliophorum von Marenzeller, 1886 
Sarcophyton trocheliophorum von Marenzeller, 1886; for further references see Imahara 1996: 22 and Benayahu 1997: 214.

Local occurrence. Yonaguni Is., Higawa, $10 \mathrm{~m}, 10$ November 1992 (ZMTAU Co 28450, 3 specimens; 28494); 12 m (ZMTAU Co 28458), Nurugan, 15 m, 11 November 1992 (ZMTAU Co 28456); Hatoma Is., northern side, $6 \mathrm{~m}$, 3 July 1993 (ZMTAU Co 28492); Ishigaki Is., Hirakubo Cape, 13 m, 4 July 1993 (ZMTAU Co 28493).

Fied notes. Sporadic.

Geographical distribution. Widespread in the Indo West-Pacific reefs.

Genus Sinularia May, 1898

Sinularia brassica May, 1898

Sinularia brassica May, 1898; for synonymy see Benayahu et al. 1998.

Local occurance. Kohama Is., Yonara, $2 \mathrm{~m}, 15$ November 1992 (ZMTAU Co 28478).

Field notes. Rare.

Geographical distribution. Widespread in the Indo West-Pacific reefs.

Sinularia ceramensis Verseveldt, 1977

Sinularia ceramensis Verseveldt, 1977: 304-307; 1980: 8 (listed only).

Local occurrence. Ishigaki Is., Masa-guchi, $8 \mathrm{~m}, 17$ November 1992 (ZMTAU Co 28631).

Field notes. Rare.

Geographical distribution. Moluccas, a new record for the Ryukyu Archipelago.

Sinularia compressa Tixier-Durivault, 1945

Sinularia compressa Tixier-Durivault, 1945; for references see Benayahu 1997: 215.

Local occurrence. Kohama Is., Yonara, $2 \mathrm{~m}, 15$ November 1992 (ZMTAU Co 29253, 2 specimens). Field notes. Rare.

Geographical distribution. Red Sea, Guam, a new record for the Ryukyu Archipelago.

Sinularia erecta Tixier-Durivault, 1945

Sinularia erecta Tixier-Durivault, 1945; for references see Benayahu 1997: 221.

Local occurrence. Ishigaki Is., off Taketomi, $10 \mathrm{~m}$, 14 November 1992 (ZMTAU Co 28633), Hirakubo
Cape, 10 m, 4 July 1993 (ZMTAU Co 28622);

Yonaguni Is., Higawa, 10 m, 10 November 1992 (ZMTAU Co 28517, 6 specimens).

Field notes. Rare.

Geographical distribution. Red Sea, Madagascar, Sodwana Bay (South Africa), Mozambique, Guam, a new record for the Ryukyu Archipelago.

Sinularia flexibilis (Quoy \& Gaimard, 1833)

Sinularia flexibilis (Quoy \& Gaimard, 1833), for synonymy see Verseveldt 1980: 54-58; Benayahu 1995: 106 (listed only); van Ofwegen 1996: 208 (listed only); van Ofwegen \& Vennam 1994: 139, 141-146; for further references see Imahara 1996: 22 .

Local occurrence. Kohama Is., Yonara, $6 \mathrm{~m}, 15$ November 1992 (ZMTAU Co 28628).

Field notes. Rare.

Geographical distribution. Widespread in the Pacific reefs.

Sinularia gibberosa Tixier-Durivault, 1970

Sinularia gibberosa Tixier-Durivault, 1970; for references see Benayahu 1997: 223-224.

Local occurrence. Kohama Is., Yonara, 6 m, 15 November 1992 (ZMTAU Co 28627).

Field notes. Rare.

Geographical distribution. Ceylon, Vietnam, Nias Is., Seychelles, Tanzania, Ryukyu Archipelago.

Sinularia gravis Tixier-Durivault, 1970

Sinularia gravis Tixier-Durivault, 1970; for references see Benayahu 1997: 224.

Local occurrence. Yonaguni Is., Higawa, reef flat, 9 November 1992 (ZMTAU CO 28629, 28463), Akatsuchi, 22 m, 13 November 1992 (ZMTAU Co 28466); Ishigaki Is., Masa-guchi, 8 m, 17 November 1992 (ZMTAU Co 28473), Ibaruma, 5 m, 4 July 1993 (ZMTAU Co 28479, 2 specimens); Miyako Is., Hisamatsu Channel, 6 m, 18 November 1992 (ZMTAU Co 28476).

Field notes. Sporadic.

Geographical distribution. Widespread in the Pacific reefs.

Sinularia heterospiculata Verseveldt, 1970

Sinularia heterospiculata Verseveldt, 1970, for references see Verseveldt 1980: 9; van Ofwegen \& 
Vennam 1994: 138 (listed only); van Ofwegen 1996: 208 (listed only); Benayahu 1993: 6 (listed only) and Benayahu \& Schleyer 1995: 6 (listed only).

Local occurrence. Ishigaki Is., off Taketomi, $6 \mathrm{~m}$, 14 November 1992 (ZMTAU Co 28513), Hirakubo Cape, 11 m, 4 July 1993 (ZMTAU Co 28623); 6 m, 5 July 1993 (ZMTAU Co 28638).

Field notes. Rare.

Geographical distribution. Madagascar, Red Sea, Ambon, Bismarck Sea, Sodwana Bay (South Africa), Mozambique, a new record for the Ryukyu Archipelago.

\section{Sinularia humesi Verseveldt, 1968}

Sinularia humesi Verseveldt, 1968: for references see Benayahu 1997: 224-229.

Local occurrence. Ishigaki Is., off Taketomi, $5 \mathrm{~m}$, 14 November 1992 (ZMTAU Co 28514); Kohama Is. Yonara, $6 \mathrm{~m}, 15$ November 1992 (ZMTAU Co 28531, 4 specimens); Iriomote Is., Kanokawa, 18 $\mathrm{m}, 16$ November 1992 (ZMTAU Co 28526, 4 specimens), $2 \mathrm{~m}$, (ZMTAU CO 28520, 2 specimens); Hatoma Is., Southern side, $6 \mathrm{~m}, 3$ July 1993 (ZMTAU Co 28467); Northern side, $6 \mathrm{~m}$ (ZMTAU Co 28470); $8 \mathrm{~m}$ (ZMTAU Co 28465, 3 specimens).

Field notes. Sporadic.

Geographical distribution. Madagascar, Red Sea, Ambon, Bismarck Sea, Guam, a new record for the Ryukyu Archipelago.

\section{Sinularia leptoclados (Ehrenberg, 1834)}

Sinularia leptoclados (Ehrenberg, 1834); for references see Imahara 1996: 22 and Benayahu 1997: 229.

Local occurrence. Kohama Is., Yonara, $2 \mathrm{~m}, 15$ November 1992 (ZMTAU Co 28626); 5 m (ZMTAU Co 28525, 2 specimens); 7 m (ZMTAU Co 28530, 2 specimens); Iriomote Is., Kanokawa, $15 \mathrm{~m}, 16$ November 1992 (ZMTAU Co 28519); Ishigaki Is., Hirakubo Cape, 14 m, 5 July 1993 (ZMTAU Co 28630); Yonaguni Is., Nurugan, 30 m, 11 November 1992 (ZMTAU Co 28505); Miyako Is., Hisamatsu Channel, $14 \mathrm{~m}, 18$ November 1992 (ZMTAU Co 28515, 2 specimens).

Field notes. Sporadic.

Geographical distribution. Widespread in the IndoPacific reefs.

Sinularia lochmodes Kolonko, 1926
Sinularia lochmodes Kolonko, 1926; for synonymy see Verseveldt 1980: 80-83; van Ofwegen \& Vennam 1991: 144 (listed only); van Ofwegen 1996: 208 (listed only); van Ofwegen \& Vennam 1994: 138 (listed only); for further references see Imahara 1996: 22 .

Local occurrence. Kohama Is., Yonara, $2 \mathrm{~m}, 15$ November 1992 (ZMTAU Co 28634); Miyako Is., Kuro Buoy, 7 m, 19 November 1992 (ZMTAU Co 28506, 3 specimens).

Field notes. Rare.

Geographical distribution. Philippines, Great Barrier Reef, Indonesia, Ambon, New Caledonia, Vietnam, Ryukyu Archipelago, Laccadives.

\section{Sinularia mollis Kolonko, 1928}

Sinularia mollis Kolonko, 1928; for synonymy see Verseveldt 1980: 92-94; Verseveldt \& Benayahu 1983: 4 (listed only); for further references see Imahara 1996: 22.

Local occurrence. Kohama Is., Yonara, $2 \mathrm{~m}, 15$ November 1992 (ZMTAU Co 28509); Iriomote Is., Kanokawa, 1 m, 16 November 1996 (ZMTAU Co 28511); Hatoma Is., southern side, $7 \mathrm{~m}$, 3 July 1993 (ZMTAU Co 28635, 2 specimens); Ishigaki Is., Hirakubo Cape, 9 m, 4 July 1993 (ZMTAU Co 28636); Miyako Is., Kuro Buoy, 9 m, 19 November 1992 (ZMTAU Co 28524, 4 specimens).

Field notes. Rare.

Geographical distribution. Philippines, Red Sea, Ryukyu Archipelago.

Sinularia numerosa Tixier-Durivault, 1970

Sinularia numerosa Tixier-Durivault, 1970; for further references see Imahara 1996: 22 and Benayahu 1997: 230.

Local occurrence. Yonaguni Is., Off Sonai, $9 \mathrm{~m}, 12$ November 1992 (ZMTAU Co 28464); 12 m (ZMTAU Co 28471), 12 November 1992; Akatsuchi, 26 m, 7 July 1993 (ZMTAU Co 28469, 2 specimens); Iriomote Is., Kanokawa, 6 m, 16 November 1992 (ZMTAU Co 28474, 2 specimens); Ishigaki Is., Hirakubo Cape, 10 m, 4 July 1993 (ZMTAU Co 28475, 2 specimens).

Field notes. Sporadic.

Geographical distribution. New Caledonia, Gambier Is., Madagascar, Guam, Ryukyu Archipelago.

Sinularia ovispiculata Tixier-Durivault, 1970 
Sinularia ovispiculata Tixier-Durivault, 1970; for further reference see Verseveldt 1980: 100-101; van Ofwegen \& Vennam 1994: 138 (listed only); Benayahu 1995: 107 (listed only) and van Ofwegen 1996: 208 (listed only).

Local occurrence. Yonaguni Is., Nurugan, $1 \mathrm{~m}, 11$ November 1992 (ZMTAU Co 28521, 2 specimens). Field notes. Rare.

Geographical distribution. Vietnam, Ambon, Ryukyu Archipelago, Bismarck Sea.

Sinularia parva Tixier-Durivault, 1970

Sinularia parva Tixier-Durivault, 1970; for further reference see Verseveldt 1980: 102-103.

Local occurrence. Yonaguni Is., off Sonai, $12 \mathrm{~m}$, 12 November 1992 (ZMTAU Co 28468).

Field notes. Rare.

Geographical distribution. Vietnam, a new record for the Ryukyu Archipelago.

Sinularia polydactyla (Ehrenberg, 1834)

Sinularia polydactyla (Ehrenberg, 1834); for further references see Imahara 1996: 22 and Benayahu 1997: 237.

Local occurrence. Yonaguni Is., off Taketomi, $10 \mathrm{~m}$, 14 November 1992 (ZMTAU Co 28625); Kohama Is., Yonara, $2 \mathrm{~m}, 15$ November 1992 (ZMTAU Co 28528); 4 m (ZMTAU Co 28522); Ishigaki Is., Hirakubo Cape, 9 m, 4 July 1993 (ZMTAU Co 28637, 3 specimens); $1 \mathrm{~m}$ (ZMTAU Co 28624); Ishigaki Is., $12 \mathrm{~m}$ (ZMTAU Co 28632); Miyako Is., Kuro Buoy, 10 m, 19 November 1992 (ZMTAU Co 28527).

Field notes. Sporadic.

Geographical distribution. Widespread in the IndoPacific reefs.

\section{Sinularia querciformis (Pratt, 1903)}

Sinularia querciformis (Pratt, 1903); for further references see Imahara, 1996: 22 and Benayahu 1997: 237-238.

Local occurrence. Iriomote Is., Kanokawa $12 \mathrm{~m}$, 16 November 1992 (ZMTAU Co 28512, 3 specimens).

Field notes. Rare.

Geographical distribution. Maldives, the Malay Archipelago, New Caledonia, Red Sea, Sodwana Bay (South Africa), Ryukyu Archipelago, Bismarck
Sea, Guam.

Sinularia variabilis Tixier-Durivault, 1945

Sinularia variabilis Tixier-Durivault, 1945: for further references see Benayahu 1997: 238.

Local occurrence. Iriomote Is., Kanokawa, $10 \mathrm{~m}$, 16 November 1992 (ZMTAU Co 28523).

Field notes. Rare.

Geographical distribution. Tuamoto Is., Gambier Is., New Caledonia, Ryukyu Archipelago, Guam.

Sinularia vrijmoethi, Verseveldt, 1971

Sinularia vrijmoethi, Verseveldt, 1971; Verseveldt 1980: 10; Benayahu 1995: 107 (listed only); for further references see Imahara 1996: 22.

Local occurrence. Kohama Is., Yonara, $1 \mathrm{~m}, 15$ November 1992 (ZMTAU Co 28507, Co 28516, Co 28518); 2 m (ZMTAU Co 28477); Iriomote Is., Kanokawa, 22 m, 16 November 1992 (ZMTAU Co 28529, 4 specimens); Hatoma Is., northern side, 8 m, 3 July 1993 (ZMTAU Co 28472); Yonaguni Is., Higawa, 14 m, 10 November 1992 (ZMTAU Co 28510, 2 specimens).

Field notes. Sporadic.

Geographical distribution. Madagascar, Red Sea, Sri Lanka, Ryukyu Archipelago.

Family Briareidae Blainville, 1830

Genus Briareum Blainville, 1830

Briaeum excavatum (Nutting, 1911)

Briaeum excavatum (Nutting, 1911); Verseveldt, 1940: 32 .

Local occurrence. Kohama Is., Yonara, $2 \mathrm{~m}, 15$ November 1992 (ZMTAU 28722); Ishigaki Is., Ibaruma, 15 m, 4 July 1993 (ZMTAU Co 28432).

Field notes. Rare.

Geographical distribution. Widespread in the Pacific reefs, a new record for the Ryukyu Archipelago.

Briareum violacea (Quoy \& Gaimard, 1833)

Briareum violacea (Quoy \& Gaimard, 1833); for synonymy see Verseveldt 1960: 211-215; Benayahu 1995: 106 (listed only); van Ofwegen 1996: 207 (listed only); for further references see Imahara 1996: 19; for taxonomic assessment see Fabricius \& Alderslade 2001: 55, 156.

Material examined. Ishigaki Is., Yarabu Cape, $6 \mathrm{~m}$, 17 November 1992 (ZMTAU Co 28719, 2 specimens); Iriomote Is., Kanokawa, 12 m, 16 November 
1992 (ZMTAU Co 28703, 4 specimens); Yonaguni Is., Nurugan, 14 m, 11 November 1992 (ZMTAU Co 28720, 2 specimens).

Field notes. Rare.

Geographical distribution. New Hebrides, Ryukyu Archipelago, Bonin Is., Formosa, Philippines, Indonesia, Malay, Great Barrier Reef.

Sinularia higai spec. nov.

Material examined. Higawa, Yonaguni Is. $10 \mathrm{~m}$, 10 November 1992, part of a colony (ZMTAU Co 29328) and additional 3 parts (ZMTAU Co 29329).

Description. The holotype and the three paratypes are illustrated in Fig. 2a and Fig. 2b-d respectively. The holotype (ZMTAU Co 29328) is part of a thin encrusting colony, with a maximum cross section of $6 \times 4.5 \mathrm{~cm}$. The surface of the colony has low mounds, thus forming a flatish and rather thin capitulum. Its outer margins are slightly raised to form a low rim, up to 8 $\mathrm{mm}$ high, with some low ridges. The polyps are completely retracted into small pits with about 2-3 $\mathrm{mm}$ between centers.

The surface layer of the capitulum has a variety of sclerites (Figs. 3, 4). The clubs possess a distinct head and their handles have small warts (Fig. 3). Length of clubs is 0.14-0.23 $\mathrm{mm}$. Spindles are also present, which are $0.12^{-}$ $0.25 \mathrm{~mm}$ long, with simple tubercles prominences (Fig. 4). The longer ones with complex tubercles are transitional with the spindles of the interior of the capitulum.

The sclerites on the surface of the base of the colony are clubs, which are wider than those of the capitulum; their length is $0.11-0.21 \mathrm{~mm}$ (Fig. 5). The interior of the base contains spindles, sometimes bifurcated, up to $3.5 \mathrm{~mm}$ long (Fig. 6a), with large tubercles of $0.1 \mathrm{~mm}$ in diameter (Fig. 6b).

Color. In alcohol the specimen is light beige.

Variability. The paratypes (Fig. 2b-d) possess the same style of sclerites as the holotype. They differ from the holotype only in size.

Etymology. This species is named for Professor Tatsuo Higa, University of the Ryukyu, in appreciation of his initiative and support in conducting this survey and his remarkable contribution to the study of natural products derived from soft corals.

Remarks. There are several other species of Sinularia that are encrusting and in which the upper face of the capitulum is more or less free of lobes, similar to $S$. higai spec. nov. (see: Alderslade
\& Baxter 1987). In addition, Benayahu (1993) described $S$. schleyeri with a similar morphology. Later, a wide intraspecific variation of colony morphology was demonstrated for S. brassica May, 1898 which includes colonies that have a capitulum with small elevations and slightly infolded margins, or even an almost flat capitulum provided with small elevations (Benayahu et al. 1998: Figs. 15, 16 respectively). However, all the species mentioned above have different sclerites.

\section{Sinuaria tanakai spec. nov}

Material examined. Higawa, Yonaguni Is. $10 \mathrm{~m}$, 10 November 1992, part of a colony (ZMTAU Co 30567) and additional four parts of a colony (ZMTAU Co 305568).

Description. The holotype and the paratypes are illustrated in Fig. 7a and Fig. 7b-e respectively. The holotype (ZMTAU Co 30567) is part of an encrusting colony, with a maximum cross section of $9 \times 5 \mathrm{~cm}$. The capitulum of the colony has sparsely placed short, erect lobes. The polyps are completely retracted with about 1-2 $\mathrm{mm}$ between centers.

The surface layer of the capitulum has clubs, 0.13-0.27 mm long, with heads consisting of spiny prominences (Fig. 8). The handles are blunt-ended and bear simple tubercles. The clubs of the surface layer of the colony base are longer than those of the capitulum and the heads possess compound tubercles; some of the smaller clubs have handles broadened by accumulation of tubercles and warts (Figs. 9, 10). The length of these clubs is $0.13-0.35 \mathrm{~mm}$. The interior of the capitulum contains spindles, sometimes branched or forked at one end; length up to 3.9 $\mathrm{mm}$ (Fig. 11a), with compound tubercles 0.1 $\mathrm{mm}$ in diameter (Fig. 11b).

Color. In alcohol the specimen is light beige.

Variability. The paratypes (Fig. 7b-e) possess the same form of sclerites as the holotype. They differ from the holotype only in size.

Etymology. This species is named after Dr. Junichi Tanaka, University of the Ryukyu, in appreciation of his friendship and tremendous help during the fieldwork of this survey, and of his contribution to the study of natural products derived from soft corals.

Remarks. S. tanakai spec. nov. (ZMTAU Co 30567) and $S$. higai spec. nov. (ZMTAU Co 29238) are encrusting soft corals, both with a flatish and thin capitulum. The former has sparse, short, erect lobes and the latter some low mounds. The sclerites of these two species differ. S. erecta 


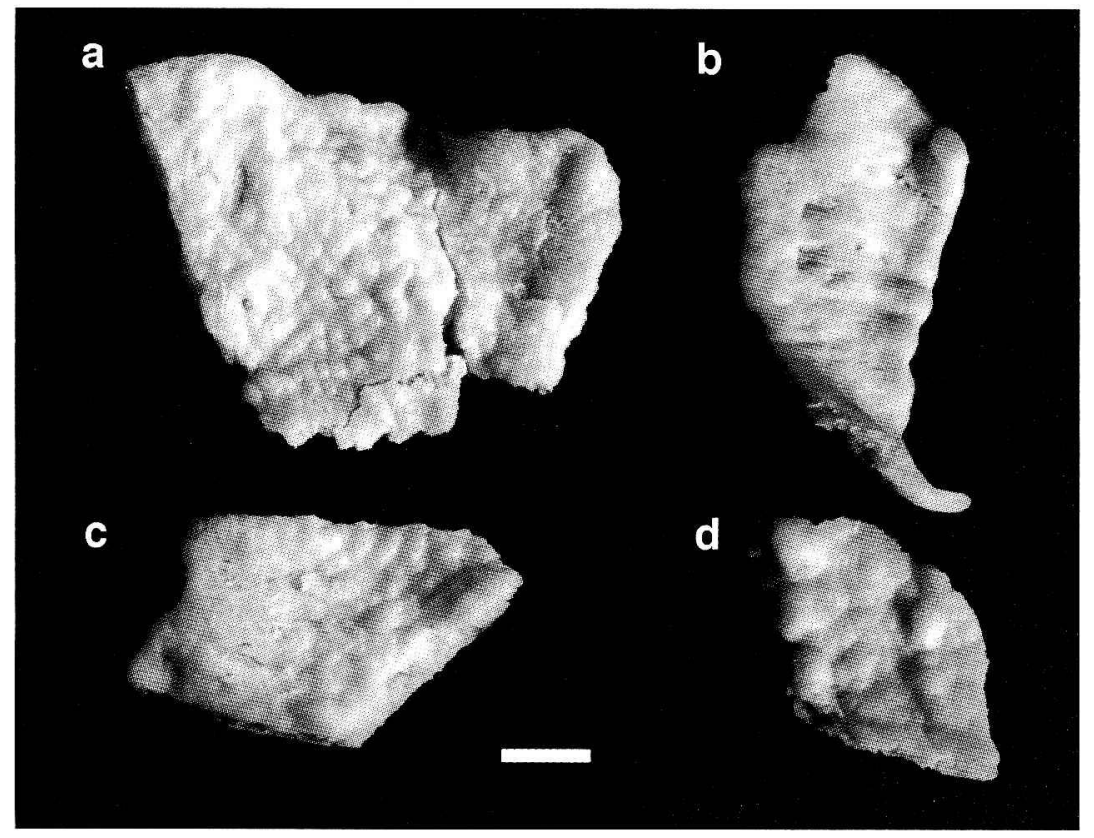

Fig 2. Sinularia higai spec. nov., a. holotype (ZMTAU Co 29328), b-d. paratyes (ZMTAU Co 29329). Scale $10 \mathrm{~mm}$.

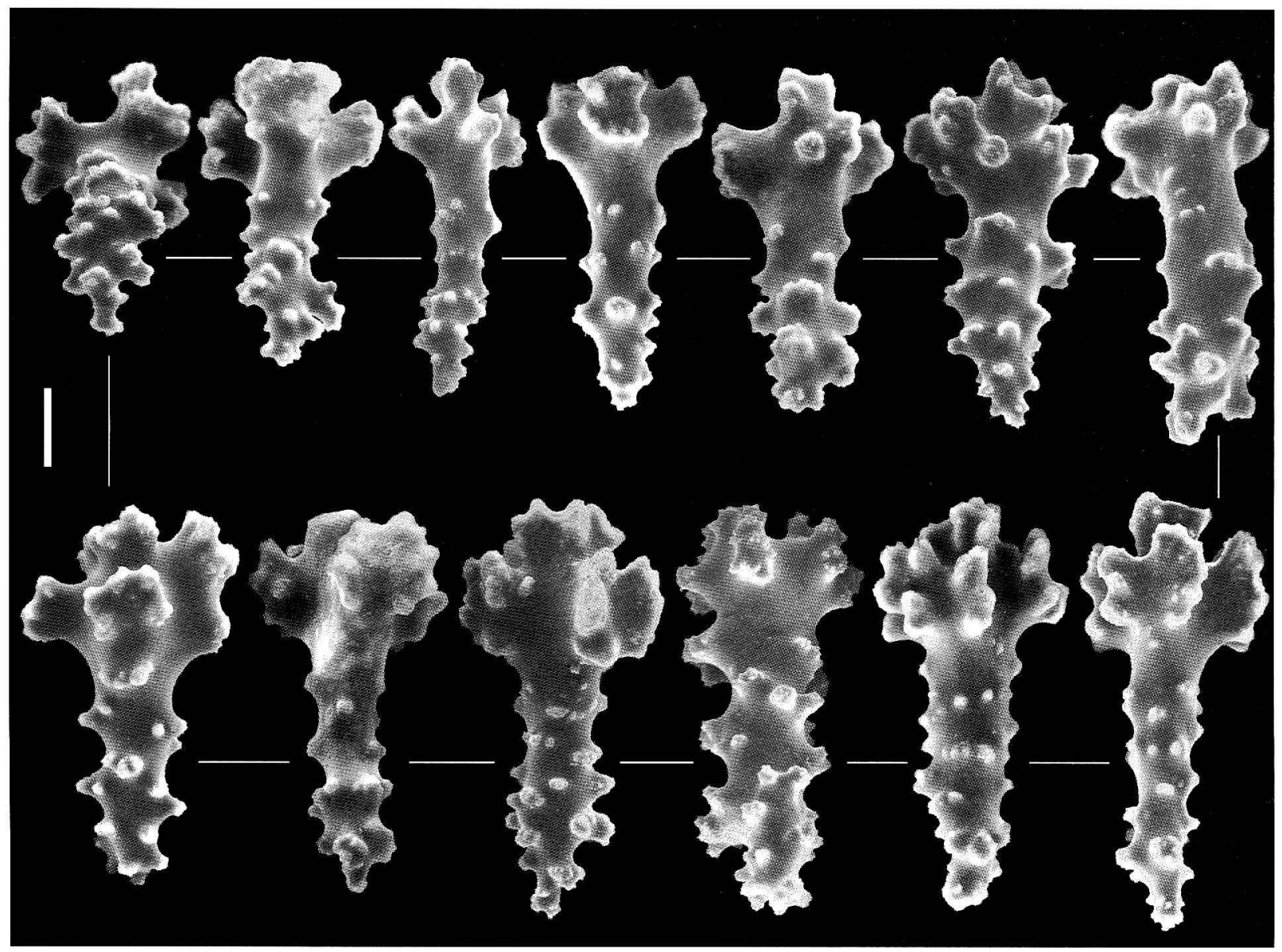

Fig 3. Sinularia higai spec. nov., holotype (ZMTAU Co 29328); clubs from the surface layer of the capitulum. Scale 0.10 $\mathrm{mm}$. 


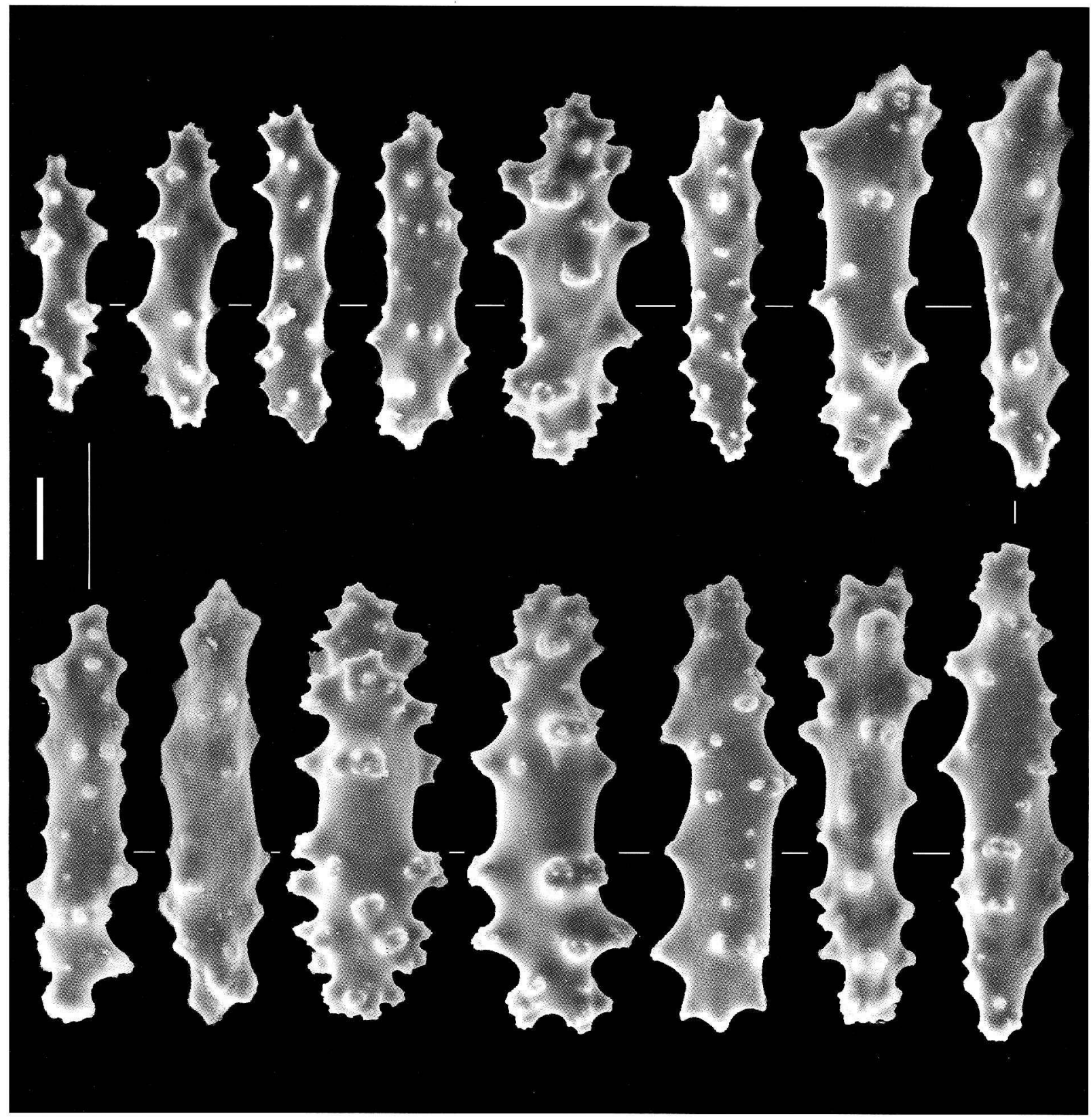

Fig 4. Sinularia higai spec. nov., holotype (ZMTAU Co 29328); spindles from the surface layer of the capitulum. Scale $0.10 \mathrm{~mm}$. 


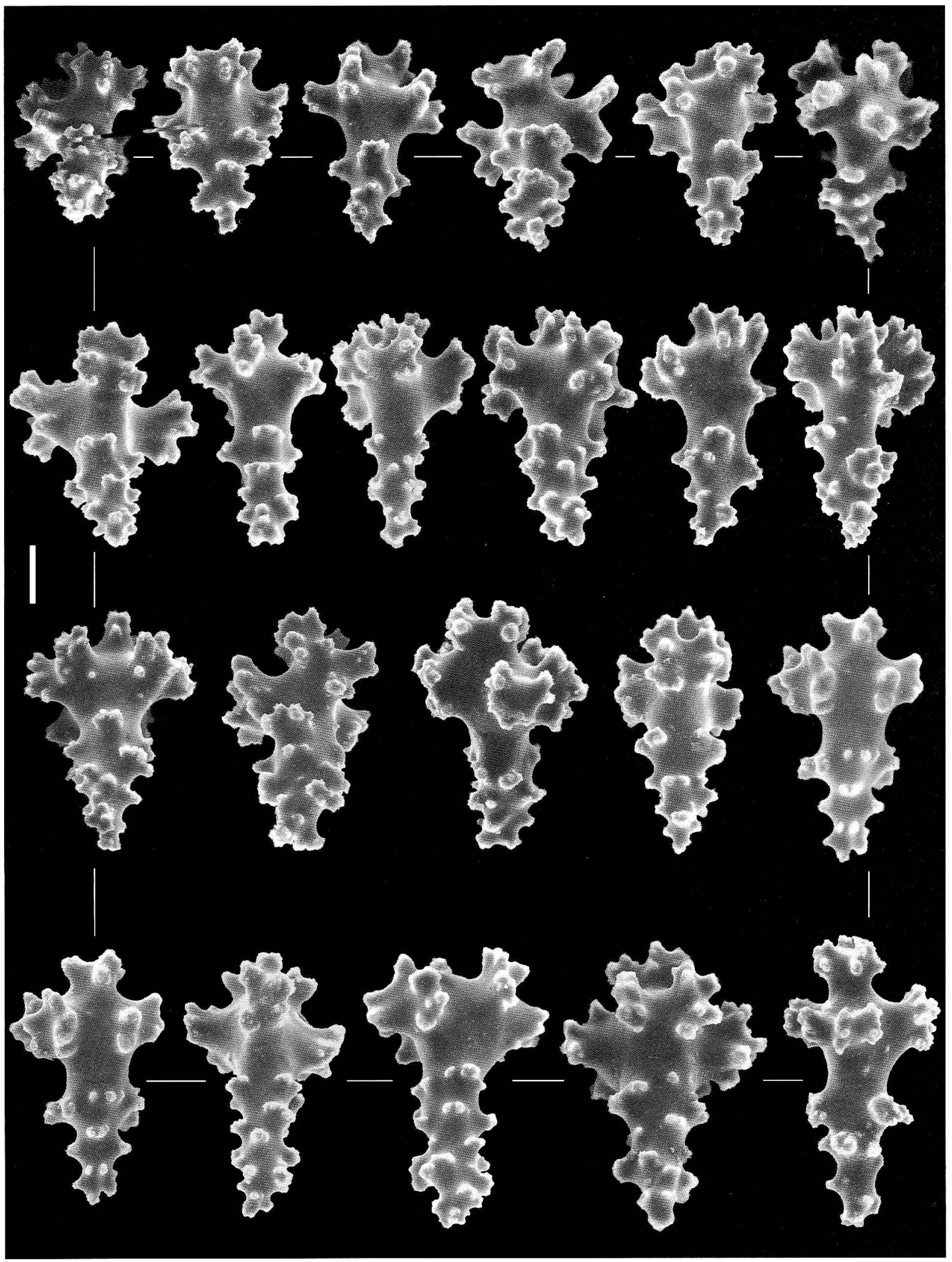

Fig 5. Sinularia higai spec. nov., holotype (ZMTAU Co 29328); clubs from the surface layer of the base. Scale $0.10 \mathrm{~mm}$. 


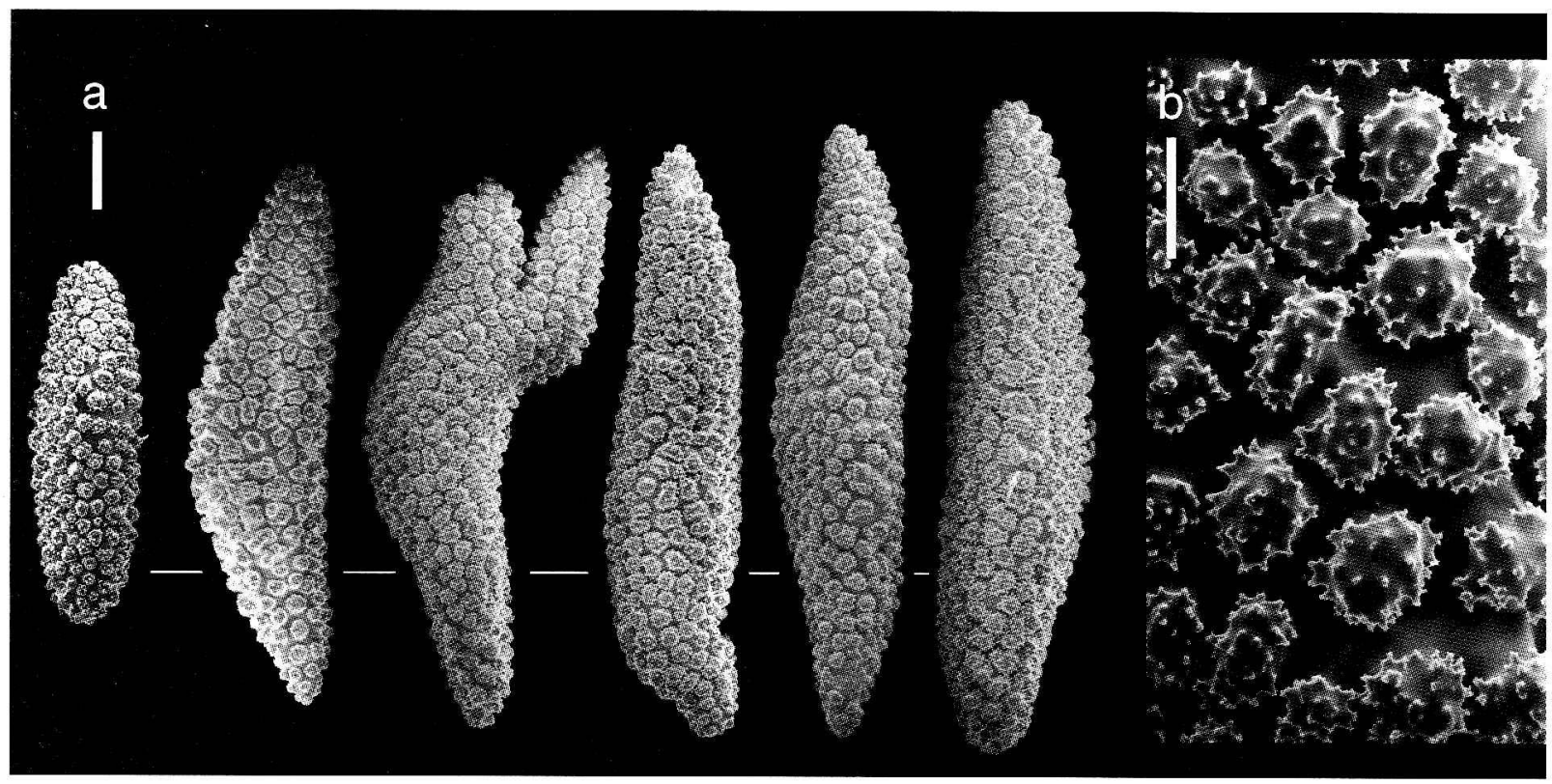

Fig 6. Sinularia higai spec. nov., holotype (ZMTAU Co 29328); a, spindles from the interior of the base; b, detail of spindle of interior of base. Scale for $6 \mathrm{a}=1 \mathrm{~mm}$, scale for $6 \mathrm{~b}=0.10 \mathrm{~mm}$.

Tixier-Durivault, 1945 also has short and erect lobes but these are densely arranged compared to S. tanakai. Furthermore, the sclerites of $S$. erecta are leptoclados-like clubs, unlike those of S. tanakai (Figs. 8-10).

Further notes. Occurrence of flat colonies with a few small-sized lobes or surface mounds among Sinularia species has been described several times. Similar flat colonies with a range of diminishing number of lobes, or even complete absence, were also described for several species of the genus Lobophytum, including $L$. depressum Tixier-Durivault, 1966, L. patulum Tixier-Durivault, 1956, L. planum Tixier-Durivault, 1970 and $L$. variatum TixierDurivault, 1957. It is suggested that such morphologies are adaptive under conditions of heavy swell and surge (see also Riegel 1995). Similarly, the type locality of both $S$. higai and $S$. tanakai spec. nov. is regularly pounded by typhoons (Tanaka personal communication). The exact significance of soft coral morphologies with a flattened capitulum, that are either free or almost free of lobes, occurring among genera that are characteristically regarded as lobate (Verseveldt \& Bayer 1988), awaits further investigation.

\section{DISCUSSION}

The overall objective of the present survey, along with the previous one (Benayahu 1995), is to investigate in detail the soft coral fauna of the Ryukyu Archipelago. These two studies indicate that soft corals are a major faunistic component on the reefs of the south Ryukyu Archipelago, including Yaeyama and Miyako island groups and Sesoko Is., totalling 56 species of the families Clavulariidae, Tubiporidae, Alcyoniidae and Briareidae. Among the 12 listed genera of these families, the present study records for the first time the genera Protodendron and Rhytisma in the Archipelago reefs. The study yielded 14 new zoogeographical records and, along with the 20 found in Sesoko Is. (Benayahu 1995), they bring to a total 34 new records, which constitute $61 \%$ of the total number of identified species for the area. Similar to the Sesoko study, the current findings reveal that species originally identified from the East China Sea (e.g., Lobophytum durum and Sinularia parva), or from other Indo-Pacific regions (e.g., Klyxum utinomii, Protodendron repens and Rhytisma fulvum fulvum) were found to be new records to the Ryukyu Archipelago. Interestingly, the current survey yielded three species, i.e.: Clavularia inflata, Tubipora musica, and Sinularia lochmodes, that appeared in Utinomi's publications (1976b, 1977b), but were not found in the Sesoko Is. survey (Benayahu 1995).

The generic composition and their respective number of species differ among the Yaeyama, Miyako and Sesoko Is. with the highest diversity found in Yaeyama Is. (Table 1). This result may 


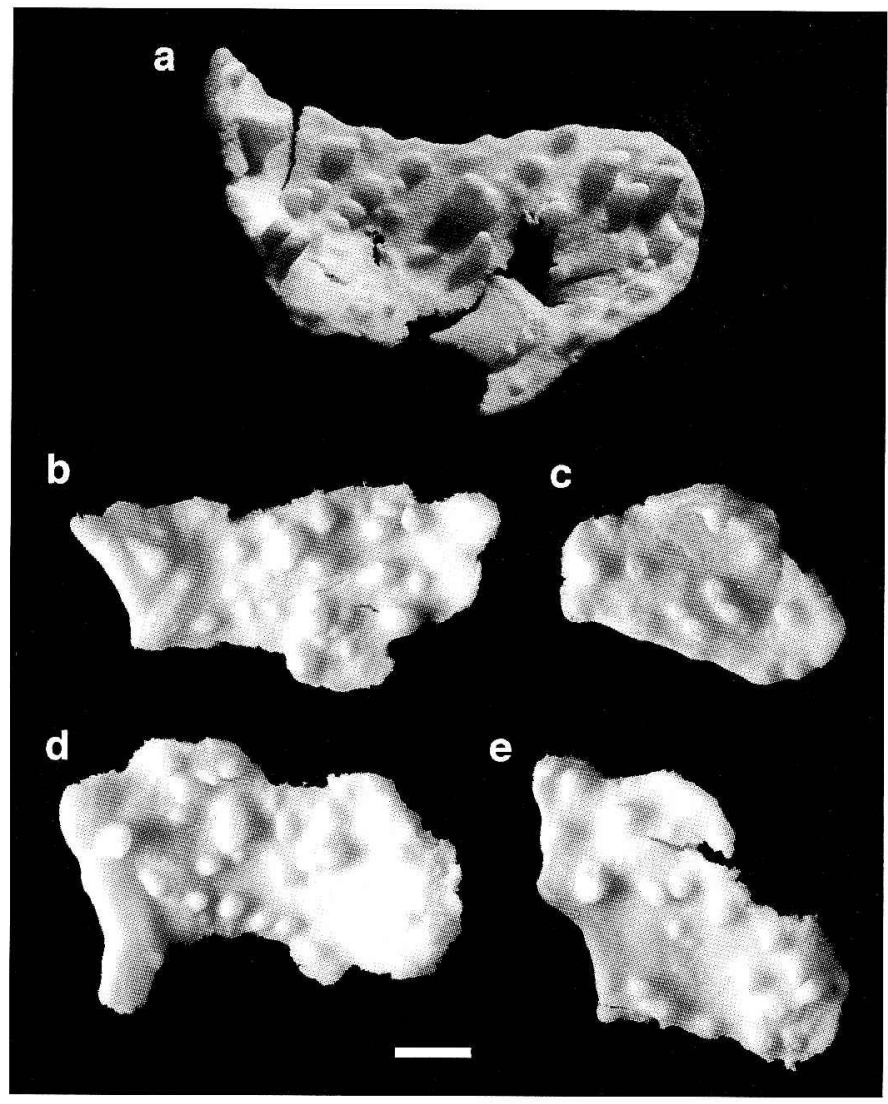

Fig 7. Sinuaria tanakai spec. nov., a holotype (ZMTAU Co 30567), b-e paratypes (ZMTAU Co 305568). Scale $10 \mathrm{~mm}$.

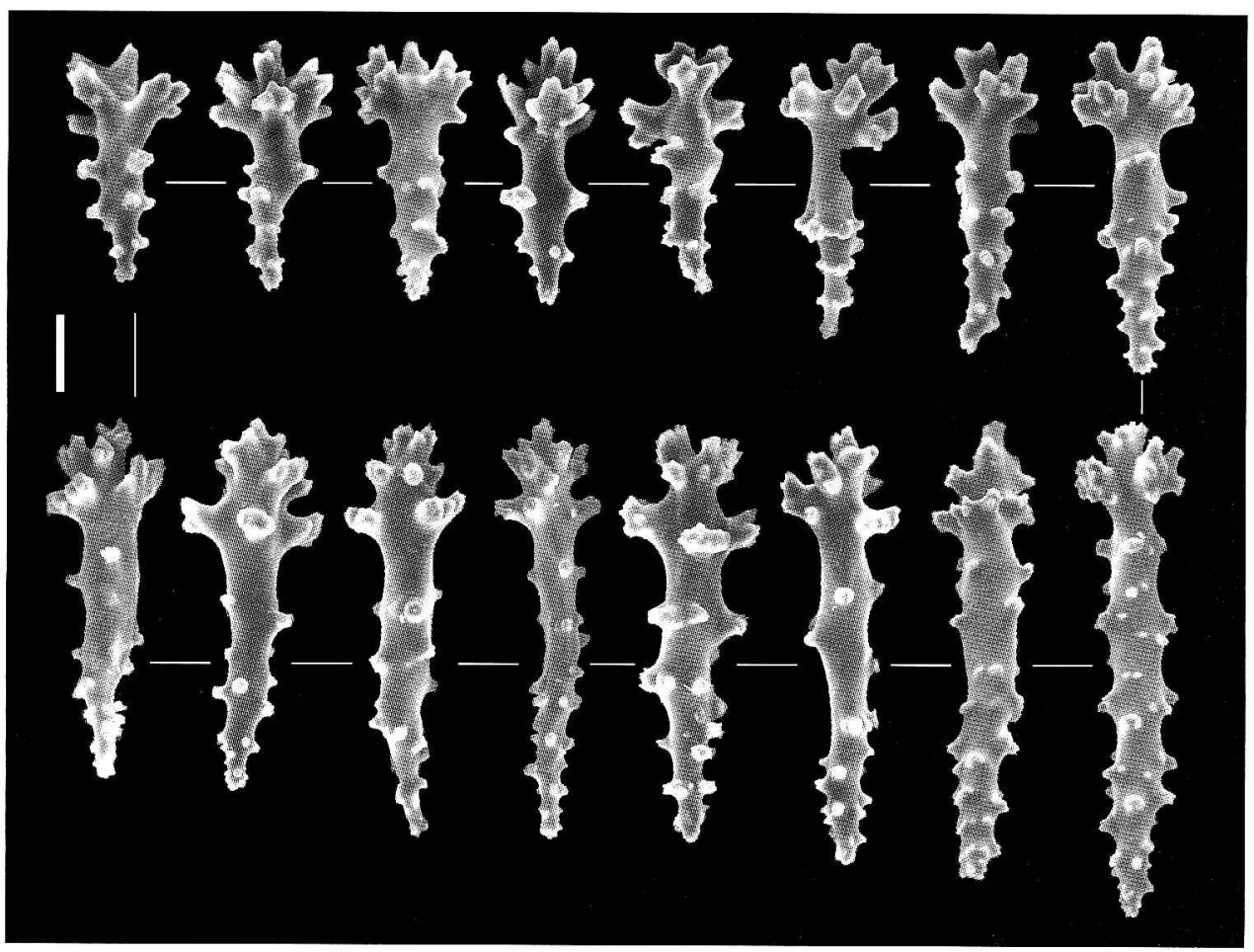

Fig 8. Sinuaria tanakai spec. nov., a holotype (ZMTAU Co 30567), clubs from the surface layer of the capitulum. Scale $0.10 \mathrm{~mm}$. 


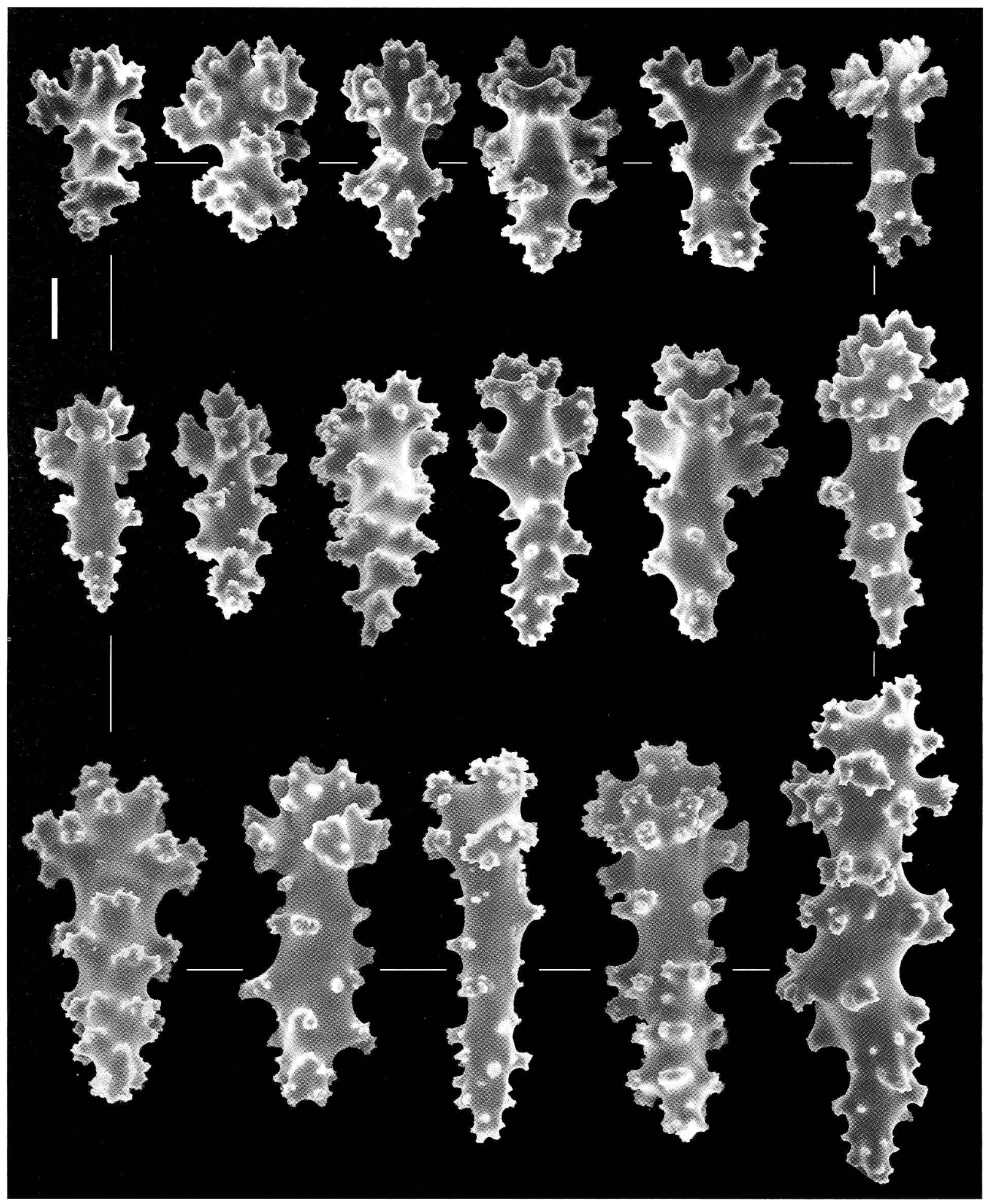

Fig 9. Sinuaria tanakai spec. nov., a holotype (ZMTAU Co 30567), clubs from the surface layer of the base. Scale 0.10 $\mathrm{mm}$. 


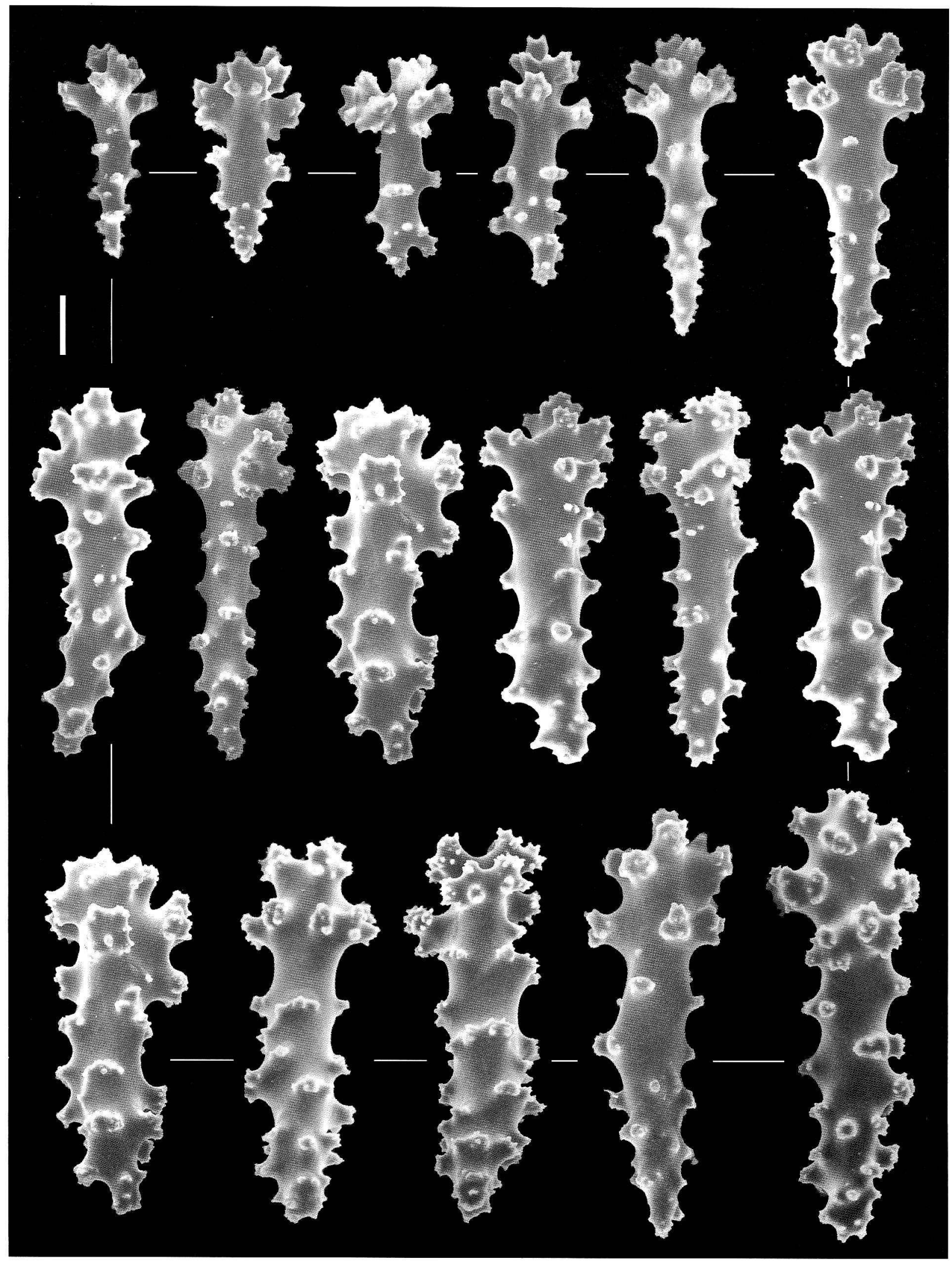

Fig 10. Sinuaria tanakai spec. nov., a holotype (ZMTAU Co 30567), clubs from the surface layer of the base. Scale 0.10 $\mathrm{mm}$. 


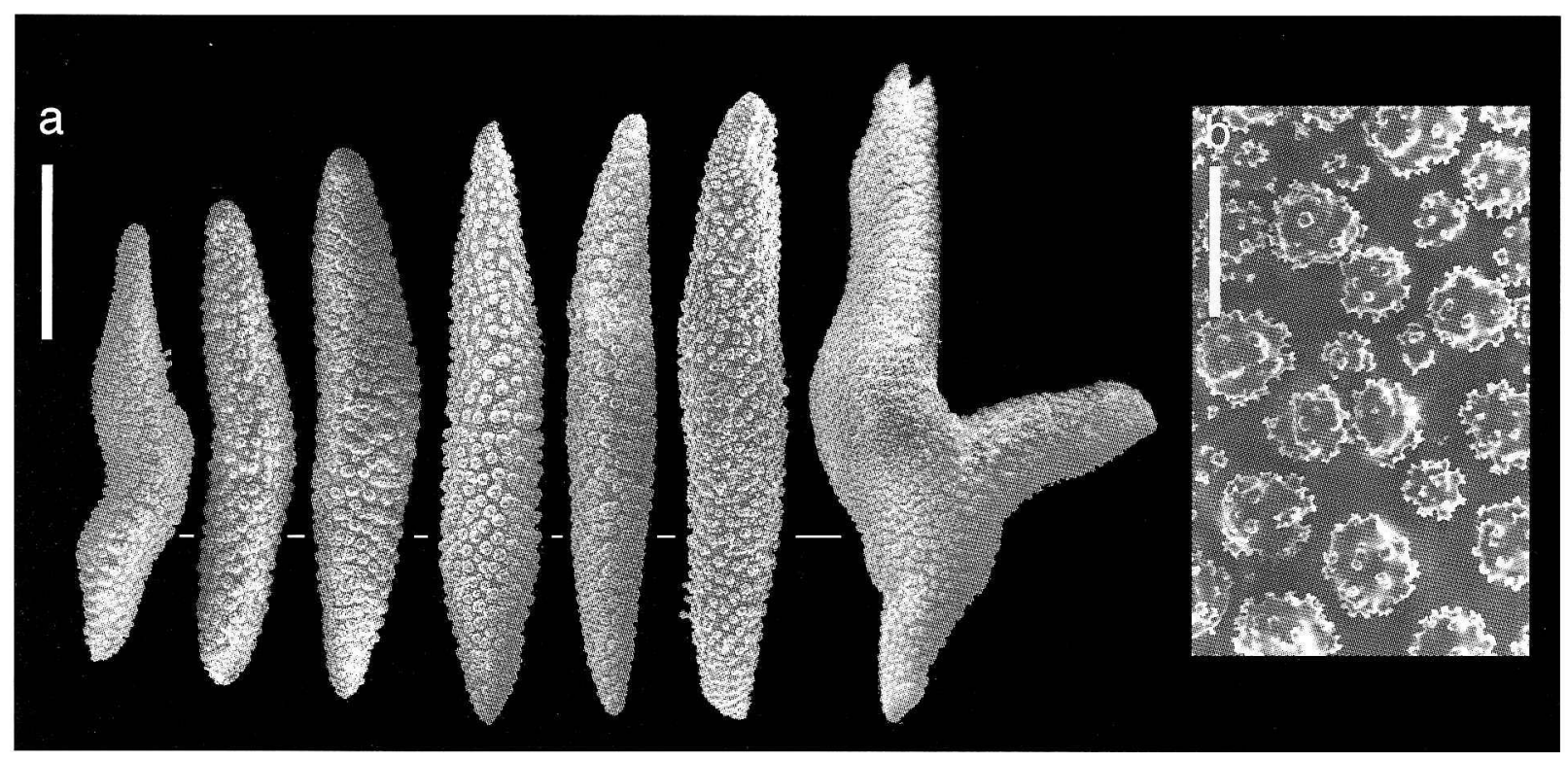

Fig 11. Sinuaria tanakai spec. nov., a holotype (ZMTAU Co 30567), a, spindles from the interior of the base; b, detail of spindle of interior of base Scale for $11 \mathrm{a}=1 \mathrm{~mm}$, scale for $11 \mathrm{~b}=0.10 \mathrm{~mm}$.

reflect the large reef area and diverse sites sur veyed there (Fig. 1). All listed genera except for Eleutherobia were recorded for the Yaeyama Is., probably resulting from the specific habitat requirements of the genus, which include mainly reef overhangs or caves, such as those found in Miyako Is. Dominance of Sinularia was evident in all islands, ranging between $33-45 \%$ of total species number. This finding is further strengthened by the discovery of the three new species i.e., S. higai and S. tanakai (this study) and $S$. yamazatoi (Benayahu 1995). Likewise, the high diversity of this genus is well known on numerous Indo-Pacific reefs (van Ofwegen in press).

The coral reefs of the Ryukyu Archipelago are threatened by natural and human induced environmental deterioration (Veron 1992). These reefs have experienced extensive coral bleaching events in recent years (e.g., Fujioka 1999; Hirose \& Hidaka 2000) and in some areas $90 \%$ of hard and soft corals bleached and died (Yamazato 1999). The widespread bleaching of the soft coral community in Sesoko Is. resulted in mass mortality and a localized near elimination of their living cover and a community-structural shift in dominance (Loya et al. 2001). Since the soft coral survey reported in the current paper was conducted prior to this bleaching event, it will be of interest to monitor the collection sites (Fig. 1) and to examine the resulting changes in the soft coral assemblages, both in terms of species composition and abundance. Such long-term moni- toring requires accurate identifaction of samples in order to document possible changes, and to avoid wrong interpretation of the results. Several studies have suggested that soft corals replace scleractinian communities after reef perturbation (references in Fabricius 1998). Thus, it is likely that recovery of the bleached Japanese reefs might be accompanied by recruitment of soft corals and a possible shift from a dominance of reef-building corals to soft coral dominated reefs. Changes in benthic communities on Okinawan reefs were induced after severe crown of thorns starfish outbreaks in the early 1970s when stony corals were replaced by soft corals, which rapidly covered large reef areas (Nishihira \& Yamazato 1974). The geographic extent of the bleaching events as well as availability of soft coral propagules in the vicinity of the devastated reefs will greatly determine the recruited species composition on these reefs. Therefore, long-term monitoring of these reefs is needed in order to evaluate the consequences of bleaching on the composition and abundance of the various soft coral taxa.

\section{ACKNOWLEDGEMENTS}

I am grateful to Prof. Tatsuo Higa, University of the Ryukyus, for his initiative to invite me to conduct this survey and for his kind hospitality. His endless efforts and support provided me with the unique opportunity to carry out the research. I would like to express my gratitude to Dr. 
Table 1. Soft coral generic composition and the respective number of species on Yaeyama and Miyako island groups and Sesoko Is., south Ryukyu Archipelago. The results for Sesoko Is. are derived from Benayahu 1995.

\begin{tabular}{|c|c|c|c|c|}
\hline & & \multicolumn{3}{|c|}{ Number of species } \\
\hline & & Yaeyama Is. & Miyako Is. & Sesoko Is. \\
\hline \multirow[t]{2}{*}{ Family } & Clavulariidae & & & \\
\hline & Clavularia & 2 & & \\
\hline \multicolumn{5}{|c|}{ Family Tubiporidae } \\
\hline & Tubipora & 1 & 1 & 2 \\
\hline \multirow[t]{9}{*}{ Family } & Alcyoniidae & & 1 & 4 \\
\hline & Klyxum & 2 & 1 & \\
\hline & Cladiella & 2 & 2 & 8 \\
\hline & Eleutherobia & & & \\
\hline & Lobophytum & 5 & & \\
\hline & Protodendron & 1 & & \\
\hline & Rhytisma & 1 & & \\
\hline & Sarcophyton & 7 & 3 & 6 \\
\hline & Sinularia & 21 & 5 & 14 \\
\hline \multicolumn{5}{|c|}{ Family Briareidae } \\
\hline & Briareum & 2 & 1 & 1 \\
\hline \multicolumn{2}{|c|}{ Total number of species } & 44 & 14 & 35 \\
\hline
\end{tabular}

Junichi Tanaka, University of the Ryukyus for his friendship and valuable assistance during the field trips. Prof. Kiyoshi Yamazato is acknowledged for his continuous support and help. I am indebted to my diving buddies: K. Ueda, S. Kouchi, C. Iha, M. Iha, D. Nagaoka and H. Nakanishi for their help. The manuscript benefited from the most constructive and critical comments of L.P. van Ofwegen who is also acknowledged for the identification of specimens of the family Briareidae and for the constructive comments on the manuscript. I wish to thank the annonimous reviewers for their helpful comments. I thank the late J.C. den Hartog, National Museum of Natural History, Leiden, The Netherlands, for his kind assistance during my visit. I wish to thank F. Scanerani for valuable assistance with the scanning microscopy, A. Shoob for photography, V. Wexsler for graphic assistance and A. Shlagman for curatorial skills. N. Paz is acknowledged for improving the style of the text. This work was part of a biotechnological study of the Ryukyu
Archipelago marine organisms, sponsored by Tropical Technology Center, Okinawa (TTC). Special thanks are due to Dr. K. Teruya, Director of the TTC, which funded my visits.

\section{REFERENCES}

Alderslade P (2000) Four new genera of soft corals (Coelenterata: Octocorallia), with notes on the classification of some established taxa. Zool Med Leiden 74: 237-249

Alderslade P, Shirwaiker P (1991) New species of soft corals (Coelenterata: Octocorallia) from the Laccadive Archipelago. The Beagle, Rec Northern Territory Mus Arts Sci 8: 189-233

Bayer FM (1995) Two species of the alconacean genus Protodendron (Octocorallia: Alcyoniidae) from the Indian Ocean off Natal. Bull Mar Sci 57: 301-312

Benayahu Y (1993) Corals of the South-west Indian Ocean I. Alcyonacea from Sodwana Bay, South Africa. Invest Rep Oceanogr Res Inst 67: 1-16

Benayahu Y (1995) Species composition of soft corals (Octocorallia, Alcyonacea) on the coral reefs of Sesoko Island, Ryukyu Archipelago, Japan. Galaxea 12: 
$103-124$

Benayahu Y (1997) A review of three alcyonacean families (Octocorallia) from Guam. Micronesica 30: 207-244

Benayahu Y, Schleyer MH (1996) Corals of the Southwest Indian Ocean III. Alcyonacea (Octocorallia) of Bazaruto Island, Mozambique, with redescription of Cladiella australis (Macfadyen, 1936) and a description of Cladiella kashmani spec. nov. Invest Rep Oceanogr Res Inst 69: 1-22

Benayahu Y, van Ofwegen LP, Alderslade P (1998) A case study of variation in two nominal species of Sinularia (Coelenterata: Octocorallia), S. brassica May, 1898 and $S$. dura (Pratt, 1903), with a proposal on their synonymy. Zool Verh Leiden 233: 277-309

Fabricius KE (1998) Reef invasion by soft corals: which taxa and which habitats? In Greenwood JG and Hall NJ, (eds), Proc. of the ACRS $75^{\text {th }}$ Anniversary Conference, Heron Island, School of Marine Science, The University of Queensland, Brisbane, pp 77-90

Fabricius K, Alderslade P (2001). Soft corals and sea fans. A comprehensive guide to the tropical shallow water genera of the central-west Pacific, the Indian Ocean and the Red Sea. Australian Institute of Marine Sciences, Queensland, Australia

Fujioka Y (1999) Mass destruction of hermatipic corals during a bleaching event in Ishigaki Island, southwest Japan. Galaxea, JSRS 1: 41-50

Hirose M, Hidaka M (2000) Reduced reproductive success in scleractinian corals that survived the 1998 bleaching in Okinawa. Galaxea, JCRS 2: 17-21

Imahara Y (1991) Reports on the Octocorallia from the Ryukyu Islands of Japan. Bull Ints Oceanic Res Develop Tokai Univ 11/12: 59-94

Imahara Y (1996) Previously recorded octocorals from Japan and adjacent seas. Precious Corals \& Octocoral Research 4-5: 17-44

Loya Y, Sakai K, Yamazato Y, Nakano H, Sambali H, van Woesik R (2001) Coral bleaching: the winners and the losers. Ecology Letters 4: 122-131

Nishihira M, Yamazato K (1974) Human interference with the coral reef community and Acanthaster infestation of Okinawa. Proc. $2^{\text {nd }}$ Int. Coral Reef Symp. Vol. 1: 577-590

Riegl B (1995) Effects of sand deposition on scleractinian and alcyonacean corals. Mar Biol 121: 517-526

Thomson JA, Dean MI (1931) The Alcyonacea of the Siboga expedition with an addendum to the Gorgonacea. Siboga-Exp Monogr 13 (d): 1-227.

Utinomi H (1976a) A review of the Japanese species of Alcyonium, with descriptions of two new species and almost forgotten rare species (Octocorallia, Alcyonacea). Publ Seto Mar Biol Lab Tech Rep 23: $191-204$

Utinomi H (1976b) Shallow-water octocorals of the Ryukyu Archipelago (Part I). Sesoko Mar Sci Lab Tech Rep 4: 1-5

Utinomi H (1977a) Shallow-water octocorals of the Ryukyu Archipelago (Part II). Sesoko Mar Sci Lab Tech Rep 5: 1-11

Utinomi H (1977b) Shallow-water octocorals of the Ryukyu Archipelago (Part III). Sesoko Mar Sci Lab Tech Rep 5: 13-34

van Ofwegen LP (1996) Octocorallia from the Bismarck Sea (part II). Zool Med Leiden 70: 207-215 van Ofwegen LP (in press). Status of knowledge of the Indo-Pacific soft coral genus Sinularia May, 1898 (Anthozoa, Octocorallia). Proc $9^{\text {th }}$ int Coral Reef Symposium, Bali, Indonesia

van Ofwegen LP, Benayahu Y (1992) Notes on Alcyonacea (Octocorallia) from Tanzania. Zool Med Leiden 66: 139-154

van Ofwegen LP, Vennam J (1991) Notes on Octocorallia from the Laccadives (SW India). Zool Meded Leiden 65: $143-154$

van Ofwegen LP, Vennam J (1994) Results of the Rumphius Biohistorical Expedition to Ambon (1990). Part 3. The Alcyoniidae (Octocorallia: Alcyonacea). Zool Med Leiden 68: 135-158

Vennam J, van Ofwegen LP (1996) Soft corals (Coelenterata: Alyonacea) from the Laccadives (SW India), with re-examination of Sinularia gravis Tixier-Durivault, 1970. Zool Med Leiden 70: 437-452

Veron JEN (1992) Conservation of biodiversity: a critical time for the hermatipic corals of Japan. Coral Reefs 11: 13-21

Verseveldt J (1940) Studies on Octocorallia of the families Briareidae, Paragorgiidae and Anthothelidae. Temminckia 5: i-v, 1-142, also as thesis, Leiden.

Verseveldt J (1960) Biological Results of the Snellius Expedition XX. Octocorallia from the Malay Archipelago (Part I). Temminckia 10: 209-250

Verseveldt J (1965) Report on the Octocorallia (Stolonifera and Alcyonacea) of the Israel South Red Sea expedition 1962, with notes on other collections from the Red Sea. Sea Fisheries Res Station, Haifa, Bull 40 (Israel South Red Sea expedition, 1962, Reports no 14: 28-48

Verseveldt J (1969) Octocorallia from North-western Madagascar (part I). Zool Verh Leiden 106: 1-38

Verseveldt J (1971) Octocorallia from North-western Madagascar (part II). Zool Verh Leiden 117: 1-73

Verseveldt J (1974) Alcyonacea (Octocorallia) from the Red Sea, with a discussion of a new Sinularia species from Ceylon. Isr J Zool 23: 1-37

Verseveldt J (1977) On two Sinularia species (Octocorallia: Alcyonacea) from the Molluccas. Zool Med Leiden 50: $303-307$

Verseveldt J (1978) Alcyonaceans (Coelenterata: Octocorallia) from some Micronesian Islands. Zool Med Leiden 53: 49-55

Verseveldt J (1980) A revision of the genus Sinularia May (Octocorallia: Alcyonacea). Zool Verh Leiden 179: $1-128$

Verseveldt J (1982) A revision of the genus Sarcophyton Lesson (Octocorallia: Alcyonacea). Zool Verh Leiden 192: 1-91

Verseveldt J (1983) A revision of the genus Lobophytum von Marenzeller (Octocorallia: Alcyonacea). Zool Verh Leiden 200: 1-103

Verseveldt J, Benayahu Y (1983) On two old and fourteen new species of Alcyonacea (Coelenterata: Octocorallia) from the Red Sea. Zool Verh Leiden 208: $1-33$

Verseveldt J, Bayer FM (1988) Revision of the genera Bellonella, Eleutherobia, Nidalia and Nidaliopsis (Octocorallia: Alcyoniidae and Nidalliidae), with descriptions of two new genera. Zool Verh Leiden 245: 1-131

Williams GC (1997) A new genus and species of 
nephtheid soft coral (Octocorallia: Alcyonacea) from the western Pacific Ocean, and a discussion of convergence with several deep-sea benthic organisms. Proc Calif Acad Sci 49: 423-437

Williams GC (2001) First record of a bioluminescent soft coral: Description of disjunct population of Eleutherobia grayi (Thomson and Dean, 1921) from Solomon Islands, with a review of bioluminescence in the Octocorallia. Proc Calif Acad Sci 52: 209-225

Yamazato K (1999) Coral bleaching in Okinawa, 1980 vs. 1998. Galaxea, JCRS, 1: 83-87

(Received: 10 January 2002/Accepted 10 April 2002) 